\title{
Protective efficacy of Ad26.COV2.S against SARS-CoV-2 B.1.351 in macaques
}

https://doi.org/10.1038/s41586-021-03732-8

Received: 15 April 2021

Accepted: 16 June 2021

Published online: 23 June 2021

Open access

Check for updates

\author{
Jingyou $\mathrm{Yu}^{1,8}$, Lisa H. Tostanoski ${ }^{1,8}$, Noe B. Mercado ${ }^{1,8}$, Katherine McMahan ${ }^{1,8}$, Jinyan Liu ${ }^{1,8}$, \\ Catherine Jacob-Dolan ${ }^{1,2,8}$, Abishek Chandrashekar ${ }^{1,8}$, Caroline Atyeo ${ }^{2,3}$, David R. Martinez ${ }^{4}$, \\ Tochi Anioke', Esther A. Bondzie', Aiquan Chang ${ }^{1,2}$, Sarah Gardner', Victoria M. Giffin', \\ David L. Hope', Felix Nampanya', Joseph Nkolola', Shivani Patel', Owen Sanborn', \\ Daniel Sellers' ${ }^{1}$, Huahua Wan', Tammy Hayes ${ }^{5}$, Katherine Bauer ${ }^{5}$, Laurent Pessaint ${ }^{6}$, \\ Daniel Valentin ${ }^{6}$, Zack Flinchbaugh ${ }^{6}$, Renita Brown ${ }^{6}$, Anthony Cook ${ }^{6}$, \\ Deandre Bueno-Wilkerson ${ }^{6}$, Elyse Teow ${ }^{6}$, Hanne Andersen ${ }^{6}$, Mark G. Lewis ${ }^{6}$, \\ Amanda J. Martinot ${ }^{5}$, Ralph S. Baric ${ }^{4}$, Galit Alter ${ }^{3}$, Frank Wegmann ${ }^{7}$, Roland Zahn $^{7}$, \\ Hanneke Schuitemaker ${ }^{7}$ \& Dan H. Barouch ${ }^{1,2,3 凶}$
}

SARS-CoV-2 variants of concern have shown increased transmissibility and pathogenicity in humans ${ }^{4,5}$, and some variants have also demonstrated partial evasion of natural and vaccine-elicited neutralizing antibodies ${ }^{1,2,6,7}$. Ad26.COV2.S is a replication-incompetent human adenovirus type 26 vector $^{8}$ that expresses a prefusion stabilized SARS-CoV-2 spike protein $(S)^{9,10}$ from the Wuhan 2019 strain of SARS-CoV-2. It was previously reported that Ad26.COV2.S demonstrated protective efficacy against SARS-CoV-2 WA1/2020 challenges in hamsters and nonhuman primates ${ }^{11-13}$, and also showed safety and immunogenicity in humans ${ }^{14,15}$. A recent phase III efficacy trial has shown that Ad26.COV2.S provided $86 \%, 88 \%$ and $82 \%$ protection against severe COVID-19 disease by day 28 after vaccination in the USA, Brazil and South Africa, respectively ${ }^{3}$.

We developed a B.1.351 challenge stock by expansion of a seed stock (BEI Resources, NR-54974) in Calu-3 cells (ATCC HTB-55). We immunized 24 rhesus macaques in 4 experimental groups $(n=6$ macaques per group) as follows: groups 1 and 3 received a sham vaccine (sham control macaques), and groups 2 and 4 received a single immunization with $5 \times 10^{10}$ viral particles of Ad26.COV2.S; after vaccination, groups 1 and 2 were challenged with the original SARS-CoV 2 strain WA1/2020, and groups 3 and 4 were challenged with the SARS-CoV-2 variant B.1.351.

\section{Ad26.COV2.S immunogenicity and cross-reactivity}

We assessed vaccine-induced antibody responses against the SARS-CoV-2 WA1/2020 strain as well as against B.1.351. Using a luciferase-based pseudovirus neutralizing antibody assay ${ }^{12,16-18}$, we found that the median neutralizing antibody titres in macaques that received Ad26.COV2.S vaccine were less than 20 at week 0 , and were 693, 561, and 155 against the WA1/2020, D614G and B.1.351 strains, respectively, in Ad26.COV2.S-vaccinated macaques at week 6 (Fig. 1a). These data show a median 4.5 -fold reduction of neutralizing antibody titres against B.1.351 as compared to WA1/2020 $(P=0.0002$, Wilcoxon

${ }^{1}$ Center for Virology and Vaccine Research, Beth Israel Deaconess Medical Center, Harvard Medical School, Boston, MA, USA. ${ }^{2} \mathrm{Harvard}$ Medical School, Boston, MA, USA. ${ }^{3}$ Ragon Institute of

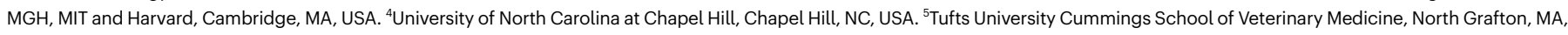
USA. ${ }^{6}$ Bioqual, Rockville, MD, USA. ${ }^{7}$ Janssen Vaccines \& Prevention, Leiden, The Netherlands. ${ }^{8}$ These authors contributed equally: Jingyou Yu, Lisa H. Tostanoski, Noe B. Mercado, Katherine McMahan, Jinyan Liu, Catherine Jacob-Dolan, Abishek Chandrashekar. ${ }^{\bowtie}$-mail: dbarouch@bidmc.harvard.edu 

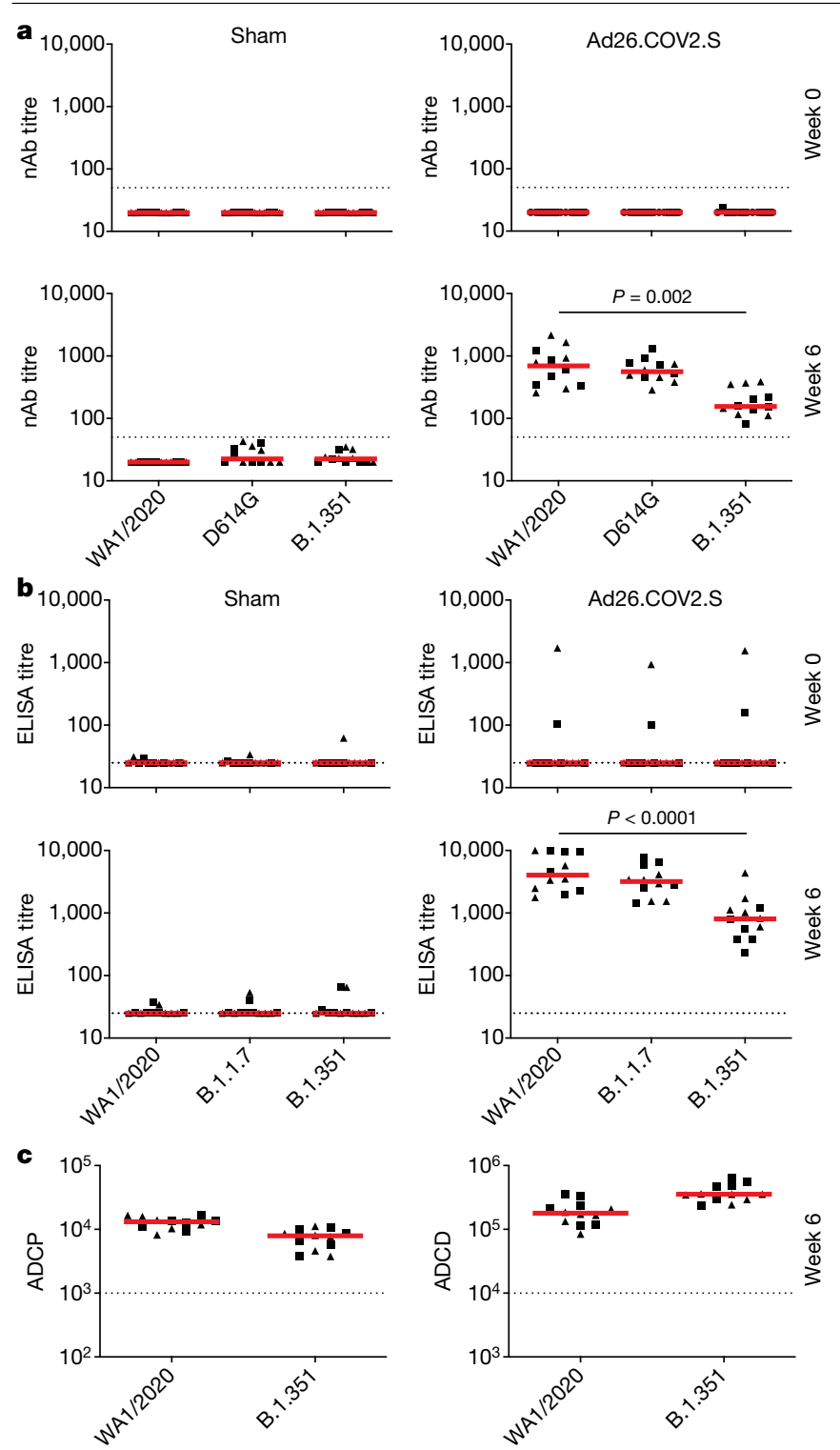

Fig. 1 Antibody responses in vaccinated rhesus macaques. a, Pseudovirus neutralizing antibody (nAb) assays against the SARS-CoV-2 WA1/2020, D614G and B.1.351 variants were assessed at week 0 (top panels) and week 6 (bottom panels) in macaques that received a single immunization of sham vaccine (left panels) or $5 \times 10^{10}$ viral particles of Ad26.COV2.S (right panels). b, RBD-specific binding antibody responses of sham control (left panels) or Ad26.COV2.Svaccinated (right panels) macaques against WA1/2020, B.1.1.7, and B.1.351 were assessed by ELISA at week 0 (top panels) and week 6 (bottom panels). c, Antibody-dependent cellular phagocytosis (ADCP) (phagocytic score) and antibody-dependent complement deposition (ADCD) (mean fluorescence intensity) were evaluated against WA1/2020 and B.1.351 at week 6. Macaques that eventually were challenged with WA1/2020 (triangles) or B.1.351 (squares) are depicted. Horizontal red bars reflect median responses. $P$ values reflect two-sided Wilcoxon rank-sum tests. Dotted lines reflect the limits of quantification of the assay. $n=24$ independent samples (12 sham and 12 Ad26.COV2.S).

rank-sum test). Live-virus neutralizing antibody assays ${ }^{19}$ showed a greater reduction of neutralizing antibody titres against B.1.351 (Extended Data Fig. 1).

Median receptor-binding domain (RBD)-specific enzyme-linked immunosorbent assay (ELISA) titres in macaques that received Ad26. COV2.S vaccine were less than 25 at week 0 , and were $4,050,3,186$ and 805 against the WA1/2020, B.1.1.7 and B.1.351 strains, respectively, in
Ad26.COV2.S-vaccinated macaques at week 6 (Fig. 1b). These data show a median 5.0-fold reduction of RBD-specific ELISA titres against B.1.351 as compared to WA1/2020 ( $P<0.0001$, Wilcoxon rank-sum test). We also used an electrochemiluminescence assay (ECLA) ${ }^{20}$ to evaluate $S$ and RBD-specific binding antibody responses to WA1/2020, B.1.1.7, P.1. and B.1.351 (Extended Data Fig. 2). Similar to the ELISA titres, median RBD-specific ECLA responses were reduced against P.1 and B.1.351 as compared to WA1/2020 at week 6, whereas we observed a smaller effect with S-specific ECLA responses. Antibody-dependent cellular phagocytosis and antibody-dependent complement deposition responses ${ }^{21}$ were more comparable against WA1/2020 and B.1.351 than were ELISA titres or ECLA responses (Fig. 1c).

We assessed S-specific cellular immune responses using pooled peptide IFN $\gamma$ enzyme-linked immunospot (ELISPOT) assays in peripheral blood mononuclear cells at week 4. ELISPOT responses were comparable among the WA1/2020, B.1.351, B.1.1.7, P.1 and CAL.20C strains, with no evidence of decreased responses against the variants (Fig. 2a). We also evaluated S-specific $\mathrm{CD}^{+}$and $\mathrm{CD} 4^{+} \mathrm{T}$ cell responses using multi-parameter intracellular cytokine staining assays at week 6 (Supplementary Fig. 1). IFN $\gamma \mathrm{CD}^{+}$and $\mathrm{CD}^{+}{ }^{+} \mathrm{T}$ cell responses were comparable among the WA1/2020, B.1.351, B.1.1.7, P.1 and CAL.20C strains (Fig. 2b). Similarly, IFN $\gamma$ central memory CD $28^{+} \mathrm{CD} 95^{+} \mathrm{CD} 4^{+}$ and $\mathrm{CD} 28^{+} \mathrm{CD} 95^{+} \mathrm{CD} 8^{+} \mathrm{T}$ cell responses were comparable across these variants (Fig. 2c). These data show that S-specific cellular immune responses were comparable for these SARS-CoV-2 variants.

\section{Homologous and heterologous SARS-CoV-2 challenges}

We challenged all macaques at week 6 with a $5 \times 10^{5} 50 \%$ tissue culture infectious dose $\left(\mathrm{TCID}_{50}\right.$ ) of SARS-CoV-2 WA1/2020 ${ }^{12,16,17,22}$ or B.1.351 by the intranasal and intratracheal routes. We assessed viral loads in bronchoalveolar lavage (BAL) and nasal swabs by reverse-transcription PCR (RT-PCR) specific for subgenomic mRNA (sgRNA), which is believed to measure replicating virus ${ }^{16,23,24}$. All sham control macaques were infected and showed higher median peak sgRNA of 6.16 (range of 4.936.80) $\log _{10}$ (sgRNA copies per $\mathrm{ml}$ ) in BAL for B.1.351, as compared to 4.80 (range of 4.70-5.52) $\log _{10}$ (sgRNA copies per ml) for WA1/2020 (Fig. 3a). By contrast, vaccinated macaques demonstrated a median peak of 3.62 (range of 3.37-4.43) $\log _{10}$ (sgRNA copies per $\mathrm{ml}$ ) in BAL for B.1.351, as compared with less than 1.69 (range of $<1.69$ to 3.23 ) $\log _{10}$ (sgRNA copies per $\mathrm{ml}$ ) in BAL for WA1/2020 (Fig. 3a). Sham control macaques also showed a trend towards a higher median peak sgRNA of 5.90 (range of 4.73-6.47) $\log _{10}$ (sgRNA copies per swab) in nasal swabs for B.1.351, as compared with 5.48 (range of 4.44-6.00) $\log _{10}$ (sgRNA copies per swab) for WA1.2020 (Fig. 3b). Vaccinated macaques demonstrated a median peak of 3.57 (range of 2.41-4.21) $\log _{10}$ (sgRNA copies per swab) in nasal swabs for B.1.351, as compared with 2.64 (range of $<1.69$ to 3.89) $\log _{10}$ (sgRNA copies per swab) in nasal swabs for WA1/2020 (Fig. 3b).

B.1.351 led to higher peak viral loads, faster kinetics of viral replication and a longer duration of viral replication as compared with WA1/2020 in sham control macaques, which suggests that B.1.351 is a more stringent challenge in the macaque model. Ad26.COV2.S provided robust protection against peak viral replication for both strains, including a 3.13 and $2.54 \log$ reduction of peak sgRNA copies per $\mathrm{ml}$ in BAL for WA1/2020 and B.1.351, respectively, and a 2.84 and $2.33 \mathrm{log}$ reduction of peak sgRNA copies per swab in nasal swabs for WA1/2020 and B.1.351, respectively $(P=0.0022$ for both BAL and nasal swabs for both WA1/2020 and B.1.351, Wilcoxon rank-sum tests) (Fig. 4a). By day 4 after challenge, viral loads were undetectable in Ad26.COV2.S-vaccinated macaques after both WA1/2020 and B.1.351 challenge, whereas viral loads were positive in most sham control macaques for WA1/2020 and in all sham control macaques for B.1.351 (Fig. 4b). Ad26.COV2.S also provided similar robust protection against day 2 infectious virus titres, as assessed by TCID $_{50}$ assays (Extended Data Fig. 3). 


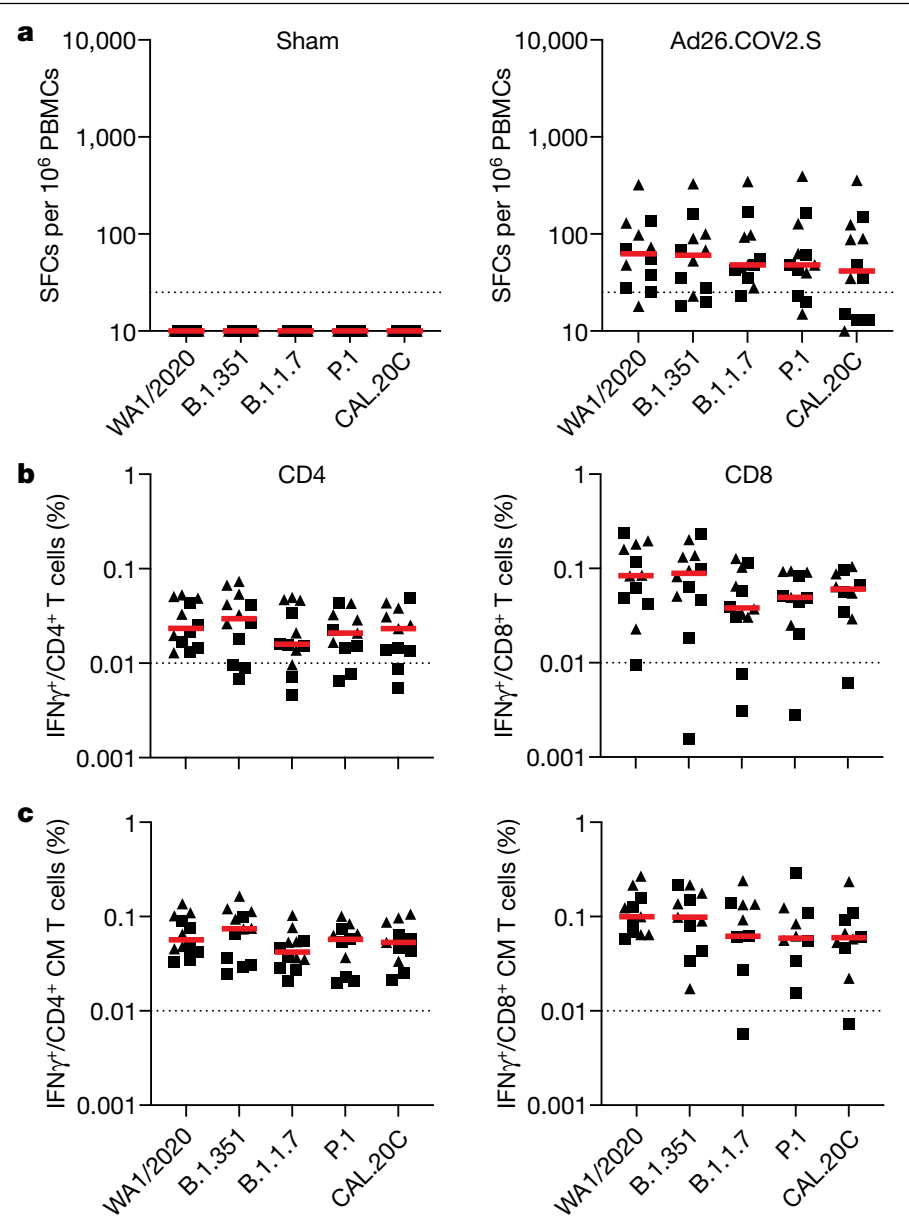

Fig. 2 | T cell responses in vaccinated rhesus macaques. a-c, Cellular immune responses to pooled S peptides of sham control (left) or Ad26.COV2.Svaccinated (right) macaques were assessed by IFN $\gamma$ ELISPOT assays at week 4 (a) and IFN $\gamma$ intracellular cytokine staining assays at week 6 (b,c) to WA1/2020, B.1.351, B.1.1.7, P.1, and CAL.20C variants. Intracellular cytokine staining assays show IFN $\gamma$ responses in $\mathrm{CD}^{+}{ }^{+}$(left) and $\mathrm{CD} 8^{+}$(right) $\mathrm{T}$ cells $(\mathbf{b})$ and $\mathrm{CD} 28^{+} \mathrm{CD} 95^{+}$ $\mathrm{CD}^{+}$(left) or $\mathrm{CD} 28^{+} \mathrm{CD} 95^{+} \mathrm{CD}^{+}$(right) central memory (CM) T cells (c).

Macaques that eventually were challenged with WA1/2020 (triangles) or B.1.351 (squares) are depicted. Horizontal red bars reflect median responses. Dotted lines reflect assay limits of quantification. $n=24$ independent samples (12 sham and 12 Ad26.COV2.S). SPCs, spot-forming cells; PBMCs, peripheral blood mononuclear cells.

\section{Correlates of protection}

On day 10 after challenge (study week 8 ), sham control macaques developed both humoral and cellular immune responses, as expected (Extended Data Figs. 4-6). In sham control macaques, WA1/2020 challenge led to higher neutralizing antibody titres to WA1/2020 than to B.1.351, whereas B.1.351 challenge led to higher neutralizing antibody titres to B.1.351 than to WA1/2020 (Extended Data Fig. 4a), and cellular responses were comparable across all strains regardless of the challenge virus (Extended Data Fig. 6), consistent with the vaccine immunogenicity data. Ad26.COV2.S-vaccinated macaques developed increased humoral and cellular immune responses after challenge. The low ELISA titres in sham control macaques probably reflect the early (day 10) time point after challenge (Extended Data Fig. 4b).

Peak $\log _{10}$ (sgRNA) in BAL (Extended Data Fig. 7) and in nasal swabs (Extended Data Fig. 8) after challenge inversely correlated with $\log _{10}$ ELISA, neutralizing antibody and ELISPOT responses at week 6 , which suggests that both antibody and $\mathrm{T}$ cell responses correlate with protection. Correlations were slightly stronger for immune responses against
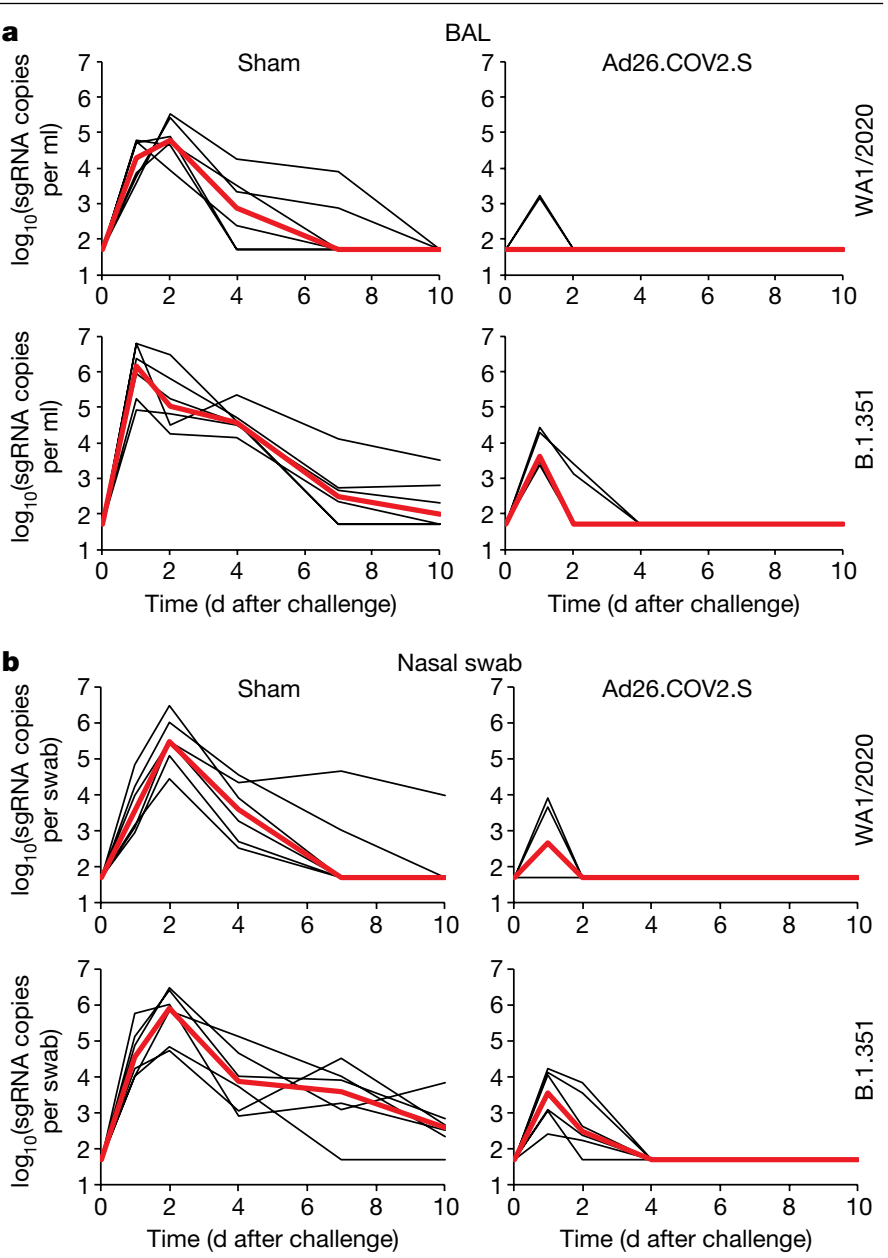

Fig. 3 | Protective efficacy after SARS-CoV-2 challenge. Rhesus macaques were challenged by the intranasal and intratracheal routes with $5 \times 10^{5} \mathrm{TCID}_{50}$ SARS-CoV-2 WA1/2020 or B.1.351. a, $\log _{10}$ (sgRNA copies per $\mathrm{ml}$ ) (limit of quantification of 50 copies per $\mathrm{ml}$ ) are shown in BAL of sham control (left) or Ad26.COV2.S-vaccinated (right) macaques after challenge with WA1/2020 (top) or B.1.351 (bottom). b, $\log _{10}$ (sgRNA copies per swab) (limit of quantification 50 copies per swab) are shown in nasal swabs of sham control (left) or Ad26.COV2.S-vaccinated (right) macaques after challenge with WA1/2020 (top) or B.1.351 (bottom). Red lines reflect median values. $n=24$ independent samples (12 sham and 12 Ad26.COV2.S).

the homologous challenge virus as compared with the heterologous challenge virus.

\section{Histopathology}

Ad26.COV2.S-vaccinated macaques demonstrated reduced lung histopathology compared with sham control macaques at necropsy on day 10 after WA1/2020 or B.1.351 challenge (Fig. 5a, b), although viral replication had largely resolved by day 10 . Sham control macaques infected with WA1/2020 and B.1.351 had histopathological lesions that were consistent with previous reports ${ }^{16}$, including focal to locally extensive interstitial pneumonia with neutrophilic and mononuclear interstitial infiltrates, alveolar syncytia, and increased numbers of alveolar macrophages. Perivascular inflammation and type II pneumocyte hyperplasia were prominent features in both groups of sham control macaques, as were multifocal regions of fibrosis (Fig. 5c, d, Extended Data Fig. 9). Ad26.COV2.S-vaccinated macaques had only rare lesions, predominantly small and focal regions of interstitial inflammation, and rare syncytia in isolated lung lobes (Fig. 5e, f, Extended Data Fig. 10). 

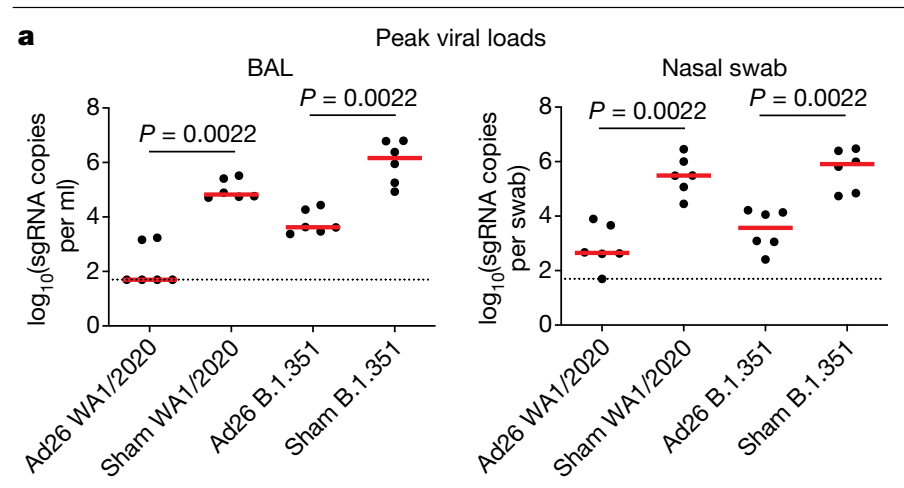

b
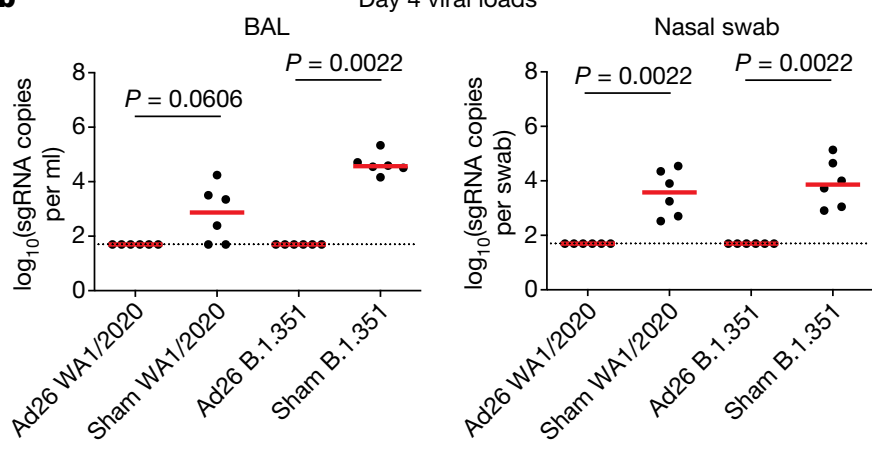

Fig. 4 | Summary of protective efficacy after SARS-CoV-2 challenge. a, b, Peak (a) and day 4 (b) viral loads in BAL (left) and nasal swabs (right) of sham control (sham) and Ad26.COV2.S-vaccinated (Ad26) macaques after challenge with WA1/2020 or B.1.351. Horizontal red bars reflect median values. $P$ values reflect two-sided Wilcoxon rank-sum tests. Dotted lines reflect the limits of quantification of the assay. $n=24$ independent samples (12 sham and 12 Ad26.COV2.S).

No evidence of eosinophilic infiltrates or enhanced respiratory disease was observed in Ad26.COV2.S-vaccinated macaques.

\section{Discussion}

It has previously been reported that Ad26.COV2.S provided robust protection against challenge with SARS-CoV-2 WA1/2020 in both rhesus macaques and hamsters ${ }^{11-13}$. In this study, we show that Ad26. COV2.S induced cross-reactive antibody and $T$ cell responses against SARS-CoV-2 variants of concern-including the B.1.351 variant, which has several mutations (including E484K) that lead to partial evasion of natural and vaccine-elicited neutralizing antibodies ${ }^{1,2,6,7}$. Binding and neutralizing antibody titres were suppressed 4-5-fold against B.1.351 as compared to WA1/2020, but Fc functional antibody responses were affected less, and $T$ cell responses were not affected at all by the SARS-CoV-2 variants. Ad26.COV2.S provided robust protection against both high-dose WA1/2020 and B.1.351 challenges. These data have important implications for the potential utility of current vaccines and inform boosting strategies against SARS-CoV-2 variants of concern.

Our data are consistent with findings in humans in a recent phase III clinical trial of Ad26.COV2.S that was conducted in the USA, Latin America (Argentina, Brazil, Chile, Colombia, Mexico and Peru) and South Africa ${ }^{3}$. Robust protection was observed in all geographical regions, with similar levels of protection against severe COVID-19 disease regardless of variant, including in the USA, in Brazil (where $69 \%$ of cases with sequence data were the P.2 variant) and in South Africa (where $95 \%$ of cases with sequence data were the B.1.351 variant). In the current study in macaques, B.1.351 infection led to a higher magnitude of and more prolonged viral replication in the upper and lower respiratory tracts than
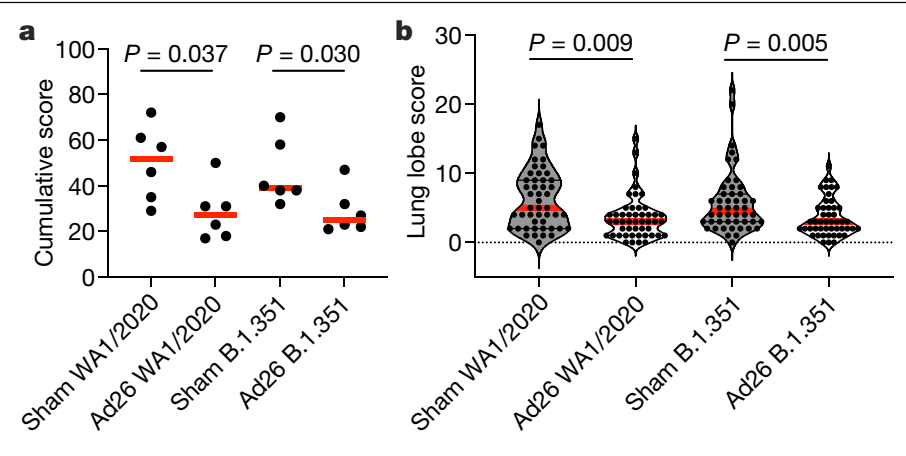

c
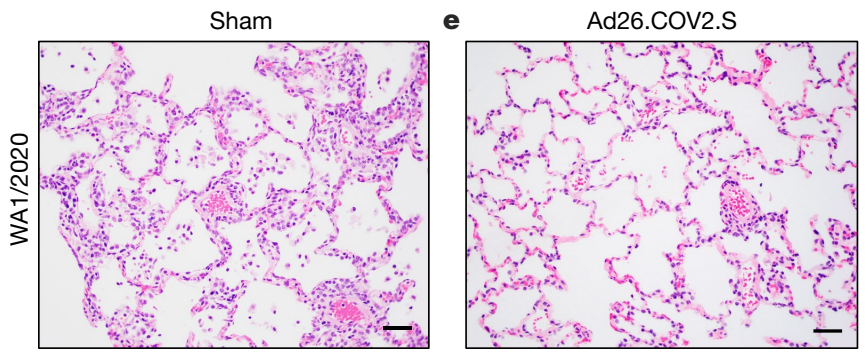

d
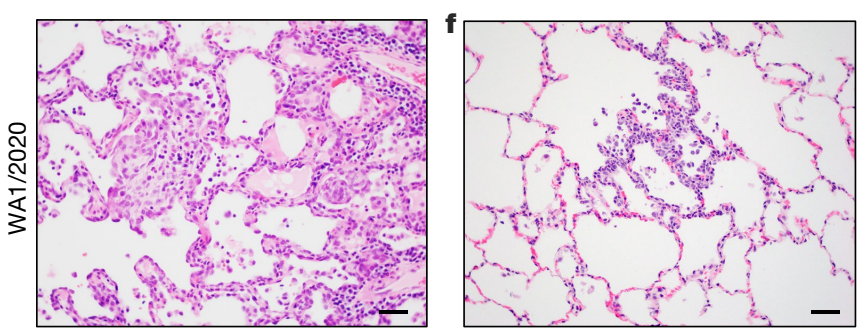

Fig. 5 | Histopathology after SARS-CoV-2 challenge. a, Cumulative histopathologic scoring of lung lesions from eight representative lung lobes from Ad26.COV2.S-vaccinated (Ad26) and sham control (sham) macaques on day 10 after challenge with WA1/2020 or B.1.351 SARS CoV-2 variants. b, Eight representative samples from cranial, middle and caudal lung lobes from the left and right lungs were evaluated from each macaque, and were scored independently for each of the following lesions: interstitial inflammation and septal thickening, interstitial infiltrate (eosinophils), interstitial infiltrate (neutrophils), hyaline membranes, interstitial fibrosis, alveolar infiltrate (macrophages), bronchoalveolar infiltrate (neutrophils), epithelial syncytia, type II pneumocyte hyperplasia, bronchi infiltrate (macrophages), bronchi infiltrate (neutrophils), bronchi (hyperplasia of bronchus-associated lymphoid tissue), bronchiolar or peribronchiolar infiltrate (mononuclear cells), perivascular infiltrate (mononuclear cells) and endothelialitis. Each feature assessed was assigned a score of: 0 , no substantial findings; 1 , minimal; 2 , mild; 3, moderate; 4, moderate to severe; 5 , marked or severe. Scores were added for all lesions across all lung lobes for each macaque, for a maximum possible score of 600 for each macaque. Horizontal red lines reflect median values. $P$ values reflect two-sided Wilcoxon rank-sum tests. c-f, Representative lung histopathology from at least eight evaluated tissues from sham control $(\mathbf{c}, \mathbf{d})$ and Ad26.COV2.S-vaccinated (e,f) macaques challenged with WA1/2020 (c,e) or B.1.351 (d,f) (on day 10 after the challenge), showing increased alveolar macrophages and thickened alveolar septa with inflammatory infiltrates and fibrosis (c), increased alveolar macrophages and epithelial syncytia within alveolar spaces, thickened and fibrotic alveolar septa with inflammatory infiltrates, focal alveolar and perivascular inflammatory infiltrates (d), focal perivascular inflammation (e) and focal expansion of alveolar septa with inflammatory infiltrates (f). Lungs evaluated were inflated or suffused with $10 \%$ formalin. In $\mathbf{c}-\mathbf{f}$, tissues were stained with haematoxylin and eosin. Scale bars, $20 \mu \mathrm{m} . n=24$ independent samples (12 sham and 12 Ad26.COV2.S) (a, b); $n=4$ representative samples ( 2 sham and 2 Ad26.COV2.S) (c-f).

did WA1/2020. Nevertheless, Ad26.COV2.S provided robust protection against both viruses, although levels of virus in BAL and nasal swabs were higher after B.1.351 challenge than after WA1/2020 challenge. 
To the best of our knowledge, this is the first report of a SARS-CoV-2 vaccine evaluated for efficacy against a SARS-CoV-2 variant of concern in macaques. Several SARS-CoV-2 vaccines have previously been reported to protect against homologous WA1/2020 challenges, but have not yet been reported against B.1.351 challenges. Our study does not define mechanistic correlates of protection against SARS-CoV-2 variants, but it has previously been reported that IgG was sufficient for protection against homologous SARS-CoV-2 challenge in macaques and that $\mathrm{CD} 8 \mathrm{~T}$ cell responses also contributed to protection if antibody titres were subprotective ${ }^{22}$.

In conclusion, Ad26.COV2.S induced cross-reactive humoral and cellular immune responses and provided robust protection against the heterologous SARS-CoV-2 variant B.1.351 in rhesus macaques. Future studies will determine whether Ad26.COV2.S, as well as other vaccines, protect against other SARS-CoV-2 variants of concern.

\section{Online content}

Any methods, additional references, Nature Research reporting summaries, source data, extended data, supplementary information, acknowledgements, peer review information; details of author contributions and competing interests; and statements of data and code availability are available at https://doi.org/10.1038/s41586-021-03732-8.

1. Wang, P. et al. Antibody resistance of SARS-CoV-2 variants B.1.351 and B.1.1.7. Nature $\mathbf{5 9 3}$ 130-135 (2021)

2. Wibmer, C. K. et al. SARS-CoV-2 501Y.V2 escapes neutralization by South African COVID-19 donor plasma. Nat. Med. 27, 622-625 (2021).

3. Sadoff, J. et al. Safety and efficacy of single-dose Ad26.COV2.S vaccine against Covid-19. N. Engl. J. Med. 384, 2187-2201 (2021).

4. Korber, B. et al. Tracking changes in SARS-CoV-2 spike: evidence that D614G increases infectivity of the COVID-19 virus. Cell 182, 812-827.e19 (2020)

5. Davies, N. G. et al. Estimated transmissibility and impact of SARS-CoV-2 lineage B.1.1.7 in England. Science 372, eabg3055 (2021).

6. Wu, K. et al. Serum neutralizing activity elicited by mRNA-1273 vaccine. N. Engl. J. Med. 384, 1468-1470 (2021).

7. Liu, Y. et al. Neutralizing activity of BNT162b2-elicited serum. N. Engl. J. Med. $\mathbf{3 8 4}$ 1466-1468 (2021).

8. Abbink, P. et al. Comparative seroprevalence and immunogenicity of six rare serotype recombinant adenovirus vaccine vectors from subgroups B and D. J. Virol. 81, 4654-4663 (2007).
B. Bos, R. et al. Ad26 vector-based COVID-19 vaccine encoding a prefusion-stabilized SARS-CoV-2 spike immunogen induces potent humoral and cellular immune responses. NPJ Vaccines 5, 91 (2020).

10. Wrapp, D. et al. Cryo-EM structure of the 2019-nCoV spike in the prefusion conformation. Science 367, 1260-1263 (2020).

11. Tostanoski, L. H. et al. Ad26 vaccine protects against SARS-CoV-2 severe clinical disease in hamsters. Nat. Med. 26, 1694-1700 (2020).

12. Mercado, N. B. et al. Single-shot Ad26 vaccine protects against SARS-CoV-2 in rhesus macaques. Nature 586, 583-588 (2020).

13. van der Lubbe, J. E. M. et al. Ad26.COV2.S protects Syrian hamsters against G614 spike variant SARS-CoV-2 and does not enhance respiratory disease. NPJ Vaccines 6, 39 (2021).

14. Sadoff, J. et al. Interim results of a phase 1-2a trial of Ad26.COV2.S Covid-19 vaccine. N. Engl. J. Med. 384, 1824-1835 (2021).

15. Stephenson, K. E. et al. Immunogenicity of the Ad26.COV2.S vaccine for COVID-19. J. Am. Med. Assoc. 325, 1535-1544 (2021).

16. Chandrashekar, A. et al. SARS-CoV-2 infection protects against rechallenge in rhesus macaques. Science 369, 812-817 (2020).

17. $\mathrm{Yu}$, J. et al. DNA vaccine protection against SARS-CoV-2 in rhesus macaques. Science 369, 806-811 (2020)

18. $\mathrm{Yu}$, J. et al. Deletion of the SARS-CoV-2 spike cytoplasmic tail increases infectivity in pseudovirus neutralization assays. J. Virol. 95, JVI.00044-21 (2021).

19. Martinez, D. R. et al. Chimeric spike mRNA vaccines protect against sarbecovirus challenge in mice. Preprint at https://doi.org/10.1101/2021.03.11.434872 (2021).

20. Jacob-Dolan, C. et al. Coronavirus-specific antibody cross reactivity in Rhesus macaques following SARS-CoV-2 vaccination and infection. J. Virol. JVI.00117-21 (2021).

21. Chung, A. W. et al. Dissecting polyclonal vaccine-induced humoral immunity against HIV using systems serology. Cell 163, 988-998 (2015).

22. McMahan, K. et al. Correlates of protection against SARS-CoV-2 in rhesus macaques. Nature 590, 630-634 (2021)

23. Wölfel, R. et al. Virological assessment of hospitalized patients with COVID-2019. Nature 581, 465-469 (2020).

24. Dagotto, G. et al. Comparison of subgenomic and total RNA in SARS-CoV-2 challenged rhesus macaques. J. Virol. 95, JVI.02370-20 (2021).

Publisher's note Springer Nature remains neutral with regard to jurisdictional claims in published maps and institutional affiliations.

Open Access This article is licensed under a Creative Commons Attribution 4.0 International License, which permits use, sharing, adaptation, distribution and reproduction in any medium or format, as long as you give appropriate credit to the original author(s) and the source, provide a link to the Creative Commons license, and indicate if changes were made. The images or other third party material in this article are included in the article's Creative Commons license, unless indicated otherwise in a credit line to the material. If material is not included in the article's Creative Commons license and your intended use is not permitted by statutory regulation or exceeds the permitted use, you will need to obtain permission directly from the copyright holder. To view a copy of this license visit http://creativecommons.org/licenses/by/4.0/.

(c) The Author(s) 2021 


\section{Methods}

No statistical methods were used to predetermine sample size. Macaques were randomized into groups. All immunological, virological and histopathological studies were performed blinded.

\section{Macaques and study design}

Twenty-four outbred Indian-origin adult male and female rhesus macaques (Macaca mulatta) (3-11 years old) were randomly allocated to groups. All macaques were housed at Bioqual. Macaques received a single immunization of $5 \times 10^{10}$ viral particles of Ad26.COV2.S $(n=12)$ or sham $(n=12)$ by the intramuscular route without adjuvant at week 0 . At week 6 , all macaques were challenged with $5 \times 10^{5} \mathrm{TCID}_{50}$ SARS-CoV-2 from strains USA-WA1/2020 (BEI Resources; NR-5228) (which was grown in VeroE6 cells and deep sequenced as previously described ${ }^{16}$ ) or B.1.351 (BEI Resources; NR-54974). The B.1.351 stock was grown in Calu-3 cells and was deep-sequenced, which confirmed the expected sequence identity with no mutations in the S greater than $2.5 \%$ frequency and no mutations elsewhere in the virus at greater than $13 \%$ frequency. Virus was administered as $1 \mathrm{ml}$ by the intranasal route $(0.5 \mathrm{ml}$ in each nare) and $1 \mathrm{ml}$ by the intratracheal route. All immunological, virological and histopathological studies were performed blinded. Animal studies were conducted in compliance with all relevant local, state and federal regulations and were approved by the Bioqual Institutional Animal Care and Use Committee.

\section{Pseudovirus-based virus neutralization assay}

The SARS-CoV-2 pseudoviruses expressing a luciferase reporter gene were generated essentially as previously described ${ }^{12,16-18}$. In brief, the packaging plasmid psPAX2 (AIDS Resource and Reagent Program), luciferase reporter plasmid pLenti-CMV Puro-Luc (Addgene) and S expressing pcDNA3.1-SARS CoV-2 S $\Delta$ CT of variants were co-transfected into HEK293T cells by lipofectamine 2000 (ThermoFisher). Pseudoviruses of SARS-CoV-2 variants were generated by using WA1/2020 strain (Wuhan/ WIV04/2019, GISAID accession identifierEPI_ISL_402124), D614G mutation, B.1.1.7 variant (GISAID accession identifier EPI_ISL_601443) or B.1.351 variant (GISAID accession identifier EPI_ISL_712096). The supernatants containing the pseudotype viruses were collected $48 \mathrm{~h}$ after transfection, and were purified by centrifugation and filtration with a $0.45-\mu \mathrm{m}$ filter. To determine the neutralization activity of the plasma or serum samples from participants, HEK293T cells expressing human ACE2 (HEK293-hACE2 cells) were seeded in 96-well tissue culture plates at a density of $1.75 \times 10^{4}$ cells per well overnight. Threefold serial dilutions of heat-inactivated serum or plasma samples were prepared and mixed with $50 \mu \mathrm{l}$ of pseudovirus. The mixture was incubated at $37^{\circ} \mathrm{C}$ for $1 \mathrm{~h}$ before adding to HEK293T-hACE2 cells. Forty-eight hours after infection, cells were lysed in Steady-Glo Luciferase Assay (Promega) according to the manufacturer's instructions. SARS-CoV-2 neutralization titres were defined as the sample dilution at which a $50 \%$ reduction in relative light units (RLU) was observed relative to the average of the virus control wells.

\section{Live virus neutralization assay}

Full-length SARS-CoV-2 WA1/2020, B.1.351 and B.1.1.7, viruses were designed to express nanoluciferase (nLuc) and were recovered via reverse genetics ${ }^{19}$. One day before the assay, Vero E6 USAMRID cells were plated at 20,000 cells per well in clear-bottom black-walled plates. Cells were inspected to ensure confluency on the day of assay. Serum samples were tested at a starting dilution of 1:20 and were serially diluted threefold up to nine dilution spots. Serially diluted serum samples were mixed in equal volume with diluted virus. Antibody-virus and virus-only mixtures were then incubated at $37^{\circ} \mathrm{C}$ with $5 \% \mathrm{CO}_{2}$ for $1 \mathrm{~h}$. After incubation, serially diluted sera and virus-only controls were added in duplicate to the cells at 75 plaque-forming units at $37^{\circ} \mathrm{C}$ with $5 \% \mathrm{CO}_{2}$. Twenty-four hours later, the cells were lysed, and luciferase activity was measured via Nano-Glo Luciferase Assay System (Promega) according to the manufacturer specifications. Luminescence was measured by a Spectramax M3 plate reader (Molecular Devices). Virus neutralization titres were defined as the sample dilution at which a $50 \%$ reduction in RLU was observed relative to the average of the virus control wells.

\section{ELISA}

WA1/2020, B.1.1.7 and B.1.351 RBD-specific binding antibodies were assessed by ELISA essentially as previously described ${ }^{12,16,17}$. In brief, 96-well plates were coated with $0.5 \mu \mathrm{g} \mathrm{ml}^{-1} \mathrm{RBD}$ protein in $1 \times$ DPBS and incubated at $4{ }^{\circ} \mathrm{C}$ overnight. After incubation, plates were washed once with wash buffer (0.05\% Tween-20 in $1 \times$ DPBS) and blocked with $350 \mu \mathrm{l}$ casein block per well for $2-3 \mathrm{~h}$ at room temperature. After incubation, block solution was discarded and plates were blotted dry. Serial dilutions of heat-inactivated serum diluted in casein block were added to wells and plates were incubated for $1 \mathrm{~h}$ at room temperature, before three further washes and a 1-h incubation with a $1 \mu \mathrm{g} \mathrm{ml}^{-1}$ dilution of anti-macaque IgG HRP (Nonhuman Primate Reagent Resource) at room temperature in the dark. Plates were then washed three times, and $100 \mu \mathrm{l}$ of SeraCare KPL TMB SureBlue Start solution was added to each well; plate development was halted by the addition of $100 \mu$ l SeraCare KPL TMB Stop solution per well. The absorbance at $450 \mathrm{~nm}$ was recorded using a VersaMax microplate reader. For each sample, ELISA endpoint titre was calculated in GraphPad Prism software, using a four-parameter logistic curve fit to calculate the reciprocal serum dilution that yields an absorbance value of 0.2 at $450 \mathrm{~nm}$. $\log _{10}$-transformed endpoint titres are reported.

\section{ECLA}

ECLA plates (MesoScale Discovery SARS-CoV-2 IgG Cat No: N05CA-1; panel 7) were designed and produced with up to nine antigen spots in each well, and assays were performed essentially as previously described $^{20}$. The antigens included were WA1/2020, B.1.1.7, P.1, and B.1.351S and RBD. The plates were blocked with $50 \mu$ l of blocker A ( $1 \%$ BSA in MilliQ water) solution for at least $30 \mathrm{~m}$ at room temperature shaking at $700 \mathrm{rpm}$ with a digital microplate shaker. During blocking, the serum was diluted 1:5,000 in diluent 100. The plates were then washed 3 times with $150 \mu$ l of the MSD kit wash buffer, blotted dry, and $50 \mu \mathrm{l}$ of the diluted samples were added in duplicate to the plates and set to shake at $700 \mathrm{rpm}$ at room temperature for at least $2 \mathrm{~h}$. The plates were again washed 3 times and $50 \mu$ of SULFO-tagged anti-human IgG detection antibody (MesoScale Discovery) diluted to $1 \times$ in diluent 100 was added to each well and incubated shaking at $700 \mathrm{rpm}$ at room temperature for at least $1 \mathrm{~h}$. Plates were then washed 3 times and $150 \mu \mathrm{l}$ of MSD GOLD read buffer $B$ was added to each well and the plates were read immediately after on a MESO QuickPlex SQ 120 machine. MSD titres for each sample are reported as RLUs, which were calculated as sample RLU minus blank RLU for each spot for each sample. The limit of detection was defined as 1,000 RLU for each assay.

\section{Fc functional antibody assays}

Fc functional profiling included the assessment of antibody-dependent monocyte phagocytosis and antibody-dependent complement deposition $^{21}$. In brief, fluorescent beads (LifeTechnologies) were coupled via carboxy-coupling, and plasma were added, allowing immune complex formation, excess antibodies were washed away, followed by the addition of THP1 monocytes, primary neutrophils or guinea pig complement, individually, respectively. The level of phagocytosis and complement deposition was assessed by flow cytometry.

\section{IFN $\gamma$ ELISPOT assay}

Pooled peptide ELISPOT assays were performed essentially as previously described ${ }^{12,16,17}$. Peptide pools consisted of 15 amino acid peptides overlapping by 11 amino acids spanning the SARS-CoV-2 S from the 
WA1/2020 strain or variant strains. ELISPOT plates were coated with mouse anti-human IFN $\gamma$ monoclonal antibody from BD Pharmigen at $5 \mu \mathrm{g}$ per well and incubated overnight at $4{ }^{\circ} \mathrm{C}$. Plates were washed with DPBS wash buffer (DPBS with $0.25 \%$ Tween-20), and blocked with R10 medium (RPMI with $10 \%$ heat-inactivated FBS with $1 \%$ of $100 \times$ penicillin-streptomycin) for $1-4 \mathrm{~h}$ at $37^{\circ} \mathrm{C}$. SARS-CoV-2 peptides (21st Century Biochemicals) were prepared and plated at a concentration of $1 \mu \mathrm{g}$ per well, and 200,000 cells per well were added to the plate. The peptides and cells were incubated for $18-24 \mathrm{~h}$ at $37^{\circ} \mathrm{C}$. All steps after this incubation were performed at room temperature. The plates were washed with ELISPOT wash buffer (11\% $10 \times$ DPBS and $0.3 \%$ Tween-20 in $11 \mathrm{MilliQ}$ water) and incubated for $2 \mathrm{~h}$ with rabbit polyclonal anti-human IFN $\gamma$ biotin from U-Cytech $\left(1 \mu \mathrm{g} \mathrm{ml}^{-1}\right)$. The plates were washed a second time and incubated for $2 \mathrm{~h}$ with streptavidin-alkaline phosphatase from Southern Biotech $\left(2 \mu \mathrm{g} \mathrm{ml}^{-1}\right)$. The final wash was followed by the addition of nitor-blue tetrazolium chloride or 5-bromo-4-chloro 3' indolyphosphate $p$-toludine salt (NBT/BCIP chromagen) substrate solution for $7 \mathrm{~min}$. The chromagen was discarded and the plates were washed with water and dried in a dim place for $24 \mathrm{~h}$. Plates were scanned and counted on a Cellular Technologies Limited Immunospot Analyzer.

\section{Intracellular cytokine staining assay}

Multi-parameter pooled-peptide intracellular cytokine staining assays were performed essentially as previously described ${ }^{12,16,17}$. Peptide pools consisted of 15 amino acid peptides overlapping by 11 amino acids spanning the SARS-CoV-2S from the WA1/2020 strain or variant strains. Then, $10^{6}$ PBMCs per well were resuspended in $100 \mu \mathrm{l}$ of R10 medium supplemented with CD49d monoclonal antibody $\left(1 \mu \mathrm{g} \mathrm{ml}^{-1}\right)$. Each sample was assessed with mock $(100 \mu$ l of R10 plus $0.5 \%$ DMSO; background control), peptide pools $\left(2 \mu \mathrm{g} \mathrm{ml}^{-1}\right)$, or $10 \mathrm{pg} \mathrm{ml}^{-1}$ phorbol myristate acetate and $1 \mu \mathrm{g} \mathrm{ml}^{-1}$ ionomycin (Sigma-Aldrich) $(100 \mu \mathrm{l}$; positive control) and incubated at $37^{\circ} \mathrm{C}$ for $1 \mathrm{~h}$. After incubation, $0.25 \mu \mathrm{l}$ of GolgiStop and $0.25 \mu \mathrm{l}$ of GolgiPlug in $50 \mu \mathrm{l}$ of R10 was added to each well and incubated at $37^{\circ} \mathrm{C}$ for $8 \mathrm{~h}$ and then held at $4{ }^{\circ} \mathrm{C}$ overnight. The next day, the cells were washed twice with DPBS, stained with near-IR live/dead dye for $10 \mathrm{~min}$ and then stained with predetermined titres of monoclonal antibodies against CD279 (clone EH12.1, BB700), CD38 (clone OKT10, PE), CD28 (clone 28.2, PE CY5), CD4 (clone L200, BV510), CD95 (clone DX2, BUV737) and CD8 (clone SK1, BUV805), for $30 \mathrm{~min}$. Cells were then washed twice with 2\% FBS in DPBS buffer and incubated for $15 \mathrm{~min}$ with $200 \mu \mathrm{l}$ of BD CytoFix/CytoPerm Fixation/Permeabilization solution. Cells were washed twice with $1 \times$ Perm Wash buffer (BD Perm/Wash Buffer 10 $\times$ in the CytoFix/CytoPerm Fixation/Permeabilization kit diluted with MilliQ water and passed through a $0.22-\mu \mathrm{m}$ filter) and stained with intracellularly with monoclonal antibodies against Ki67 (clone B56, FITC), CD69 (clone TP1.55.3, ECD), IL-10 (clone JES3-9D7, PE CY7), IL-13 (clone JES10-5A2, BV421), TNF (clone Mab11, BV650), IL-4 (clone MP4-25D2, BV711), IFN (clone B27; BUV395), CD45 (clone D058-1283, BUV615), IL-2 (clone MQ1-17H12, APC) and CD3 (clone SP34.2, Alexa 700), for $30 \mathrm{~min}$. Cells were washed twice with $1 \times$ Perm Wash buffer and fixed with $250 \mu$ of freshly prepared $1.5 \%$ formaldehyde. Fixed cells were transferred to a 96-well round-bottom plate and analysed by BD FACSymphony system. Central memory T cells were defined as $\mathrm{CD} 28^{+} \mathrm{CD} 95^{+} \mathrm{T}$ cells. Data were analysed with FlowJo v.9.9.

\section{sgRNA assay}

SARS-CoV-2 $E$ gene sgRNA was assessed by RT-PCR using primers and probes as previously described ${ }^{23,24}$. A standard was generated by first synthesizing a gene fragment of the subgenomic $E$ gene $^{23}$. The gene fragment was subsequently cloned into a pcDNA3.1+ expression plasmid using restriction site cloning (Integrated DNA Techonologies). The insert was in vitro-transcribed to RNA using the AmpliCap-Max T7 High Yield Message Maker Kit (CellScript). log dilutions of the standard were prepared for RT-PCR assays ranging from $1 \times 10^{10}$ copies to $1 \times 10^{-1}$ copies. Viral loads were quantified from BAL fluid and nasal swabs.
RNA extraction was performed on a QIAcube HT using the IndiSpin QIAcube HT Pathogen Kit according to manufacturer's specifications (Qiagen). The standard dilutions and extracted RNA samples were reverse-transcribed using SuperScript VILO Master Mix (Invitrogen) following the cycling conditions described by the manufacturer, $25^{\circ} \mathrm{C}$ for $10 \mathrm{~min}, 42^{\circ} \mathrm{C}$ for $1 \mathrm{~h}$, then $85^{\circ} \mathrm{C}$ for $5 \mathrm{~min}$. A Taqman custom gene expression assay (Thermo Fisher Scientific) was designed using the sequences targeting the $E$ gene sgRNA ${ }^{23}$. The sequences for the custom assay were as follows, forward primer, sgLeadCoV2.Fwd: CGATCTCTTGTAGATCTGTTCTC, E_Sarbeco_R: ATATTGCAGCAGTACGCACACA, E_Sarbeco_P1 (probe):VIC-ACACTAGCCATCCTTACTGCGCTTCG-MGB. These primers and probes were equally reactive for both variants. Reactions were carried out in duplicate for samples and standards on the QuantStudio 6 and 7Flex Real-Time PCR Systems (Applied Biosystems) with the thermal cycling conditions, initial denaturation at $95^{\circ} \mathrm{C}$ for $20 \mathrm{~s}$, then 45 cycles of $95^{\circ} \mathrm{C}$ for $1 \mathrm{~s}$ and $60^{\circ} \mathrm{C}$ for $20 \mathrm{~s}$. Standard curves were used to calculate sgRNA copies per ml or per swab; the quantitative assay sensitivity was 50 copies per $\mathrm{ml}$ or per swab.

\section{TCID $_{50}$ assay}

Vero TMPRSS2 cells (obtained from A. Creanga) were plated at 25,000 cells per well in DMEM with $10 \% \mathrm{FBS}$ and gentamicin, and the cultures were incubated at $37^{\circ} \mathrm{C}, 5.0 \% \mathrm{CO}_{2}$. Medium was aspirated and replaced with $180 \mu \mathrm{l}$ of DMEM with $2 \%$ FBS and gentamicin. Serial dilution of samples as well as positive (virus stock of known infectious titre) and negative (medium only) controls were included in each assay. The plates are incubated at $37^{\circ} \mathrm{C}, 5.0 \% \mathrm{CO}_{2}$ for 4 days. Cell monolayers were visually inspected for cytopathic effect. The $\mathrm{TCID}_{50}$ was calculated using the Read-Muench formula.

\section{Histopathology}

Lungs on day 10 after SARS-CoV-2 challenge were evaluated by histopathology. At the time of fixation, lungs were suffused with $10 \%$ formalin to expand the alveoli. All tissues were fixed in $10 \%$ formalin and blocks sectioned at $5 \mu \mathrm{m}$. Slides were incubated for 30-60 min at $65^{\circ} \mathrm{C}$ then deparaffinized in xylene and rehydrated through a series of graded ethanol to distilled water. Sections were stained with haematoxylin and eosin. Blinded evaluation and scoring was performed by a board-certified veterinary pathologist (A.J.M.).

\section{Statistical analyses}

Comparisons of virological, immunological and histopathological data were performed using GraphPad Prism 8.4.2 (GraphPad Software). Comparison of data between groups was performed using two-sided Wilcoxon rank-sum tests. Correlation analyses were performed using two-sided Spearman rank-correlation tests. $P$ values of less than 0.05 were considered significant.

\section{Reporting summary}

Further information on research design is available in the Nature Research Reporting Summary linked to this paper.

\section{Data availability}

All relevant data are available in the Article and its Supplementary Information. Any additional data are available from the corresponding author upon reasonable request. Source data are provided with this paper.

Acknowledgements We thank J. Muench, F. Paz, J. Yalley-Ogunro, S. O'Connor, A. Sigal, T. de Oliveira, J. Barrett, M. Gebre and M. Lifton for advice, assistance, and reagents; and Mesoscale Discovery for the ECLA kits. This project was funded in part by the Department of Health and Human Services Biomedical Advanced Research and Development Authority (BARDA) under contract HHS0100201700018C. We also acknowledge support from Janssen Vaccines \& Prevention BV, Ragon Institute of MGH, MIT and Harvard, Musk Foundation, Massachusetts 


\section{Article}

Consortium on Pathogen Readiness (MassCPR) and the National Institutes of Health (CA260476).

Author contributions D.H.B., F.W. and R.Z. designed the study. J.Y., L.H.T., N.B.M., K.M., J.L., C.J.-D., A. Chandrashekar, T.A., E.A.B., A. Chang, S.G., V.M.G., D.L.H., F.N., J.N., S.P., O.S., D.S and H.W. performed the immunological and virological assays. D.R.M. and R.S.B. performed the live virus neutralization assays. C.A. and G.A. performed the Fc functional antibody assays. L.P., D.V., Z.F., R.B., A. Cook, D.B.-W., E.T., H.A. and M.G.L. led the clinical care of the macaques F.W., R.Z. and H.S. provided the vaccine. T.H., K.B. and A.J.M. led the histopathology. D.H.B. wrote the paper with all co-authors.
Competing interests D.H.B., R.Z., F.W. and H.S. are co-inventors on provisional vaccine patents (63/121,482; 63/133,969; 63/135,182). R.Z., F.W. and H.S. are employees of Janssen Vaccines \& Prevention BV and may hold stock in Johnson \& Johnson.

Additional information

Supplementary information The online version contains supplementary material available at https://doi.org/10.1038/s41586-021-03732-8.

Correspondence and requests for materials should be addressed to $D . H . B$.

Peer review information Nature thanks Wolfgang Baumgärtner, Andrianus Boon and the other, anonymous, reviewer(s) for their contribution to the peer review of this work. Reprints and permissions information is available at http://www.nature.com/reprints. 
Sham

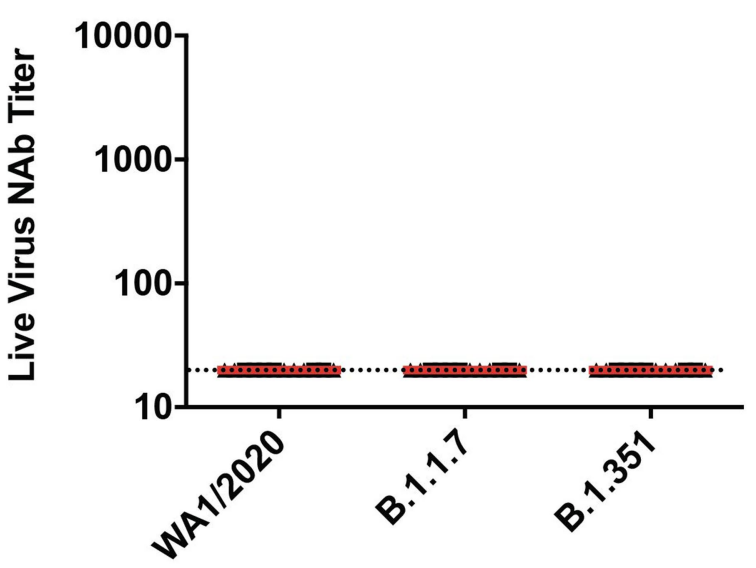

Extended Data Fig. 1 | Live virus neutralizing antibody responses in vaccinated rhesus macaques. Live virus neutralizing antibody responses against the SARS-CoV-2 WA1/2020, B.1.1.7 and B.1.351 variants were assessed at week 6 in macaques that received a single immunization of sham vaccine or
Ad26.COV2.S

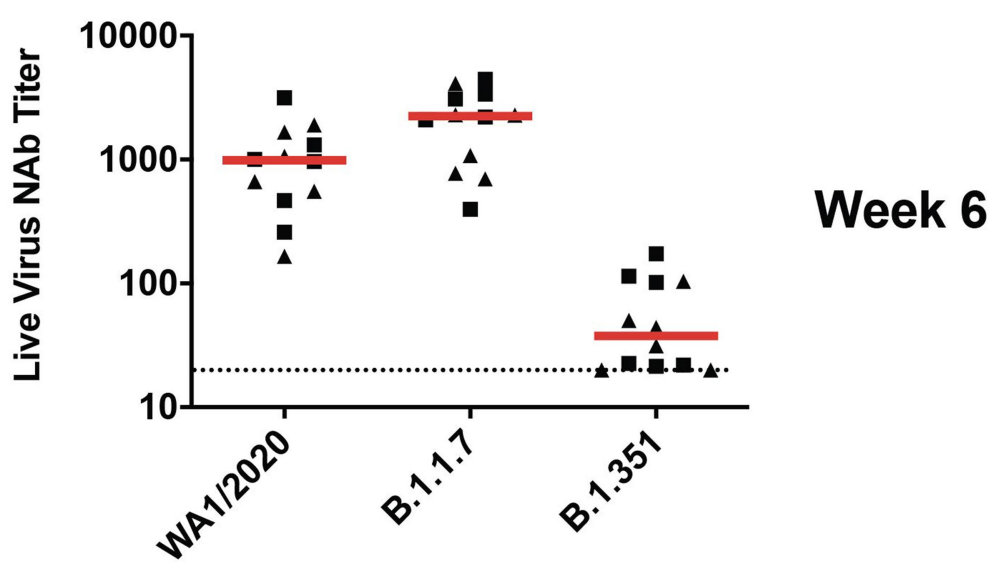

$5 \times 10^{10}$ viral particles of Ad26.COV2.S. Macaques that eventually were challenged with WA1/2020 (triangles) or B.1.351 (squares) are depicted. Horizontal red bars reflect median responses. Dotted lines reflect assay limits of quantification. $n=24$ independent samples (12 sham, 12 Ad26.COV2.S). 


\section{Article}

a

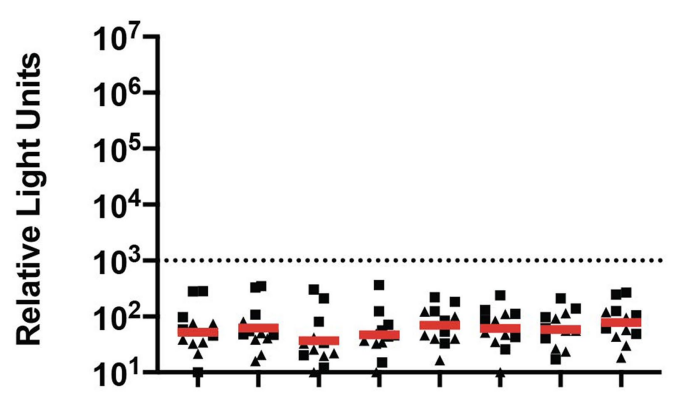

b

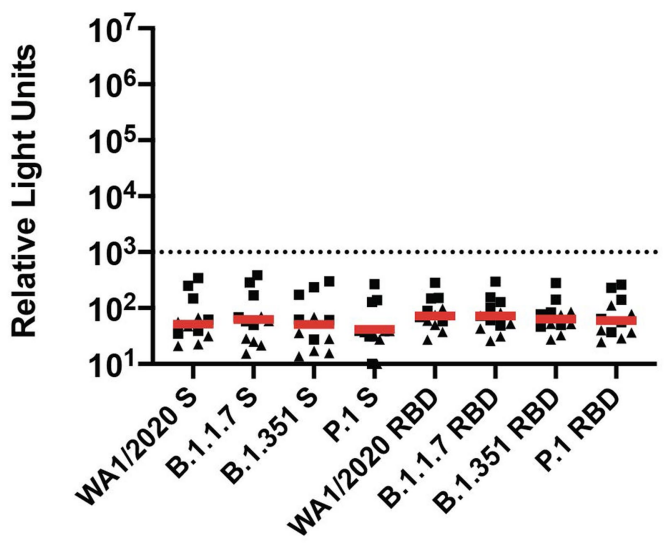

Extended Data Fig. 2 | Binding antibody responses in vaccinated rhesus macaques by ECLA. a, b, S- and RBD-specific binding antibody responses against the SARS-CoV-2 WA1/2020,B.1.1.7,B.1.351 and P.1 variants were assessed by ECLA at week 0 (a) and week $6(\mathbf{b})$ in macaques that received a
Ad26.COV2.S

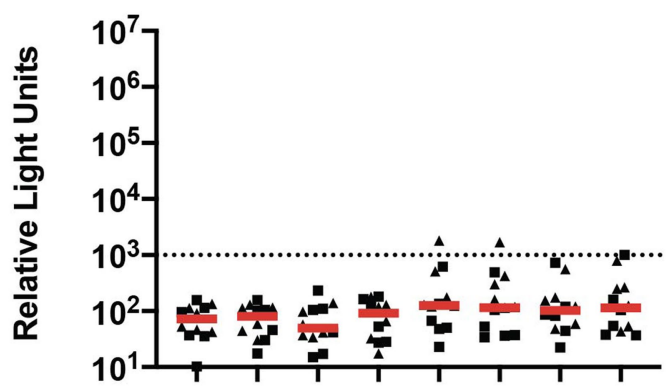

Week 0

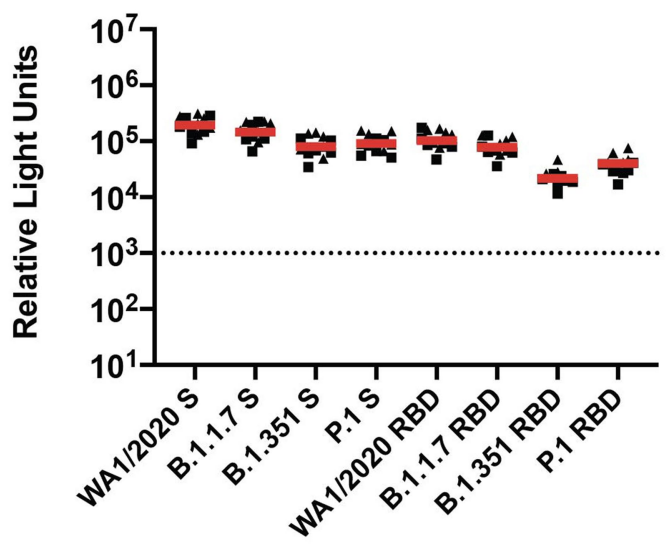

Week 6

single immunization of sham-negative control or $5 \times 10^{10}$ viral particles of Ad26. COV2.S. Macaques that eventually were challenged with WA1/2020 (triangles) or B.1.351 (squares) are depicted. Horizontal red bars reflect median responses. Dotted lines reflect assay limits of quantification. 


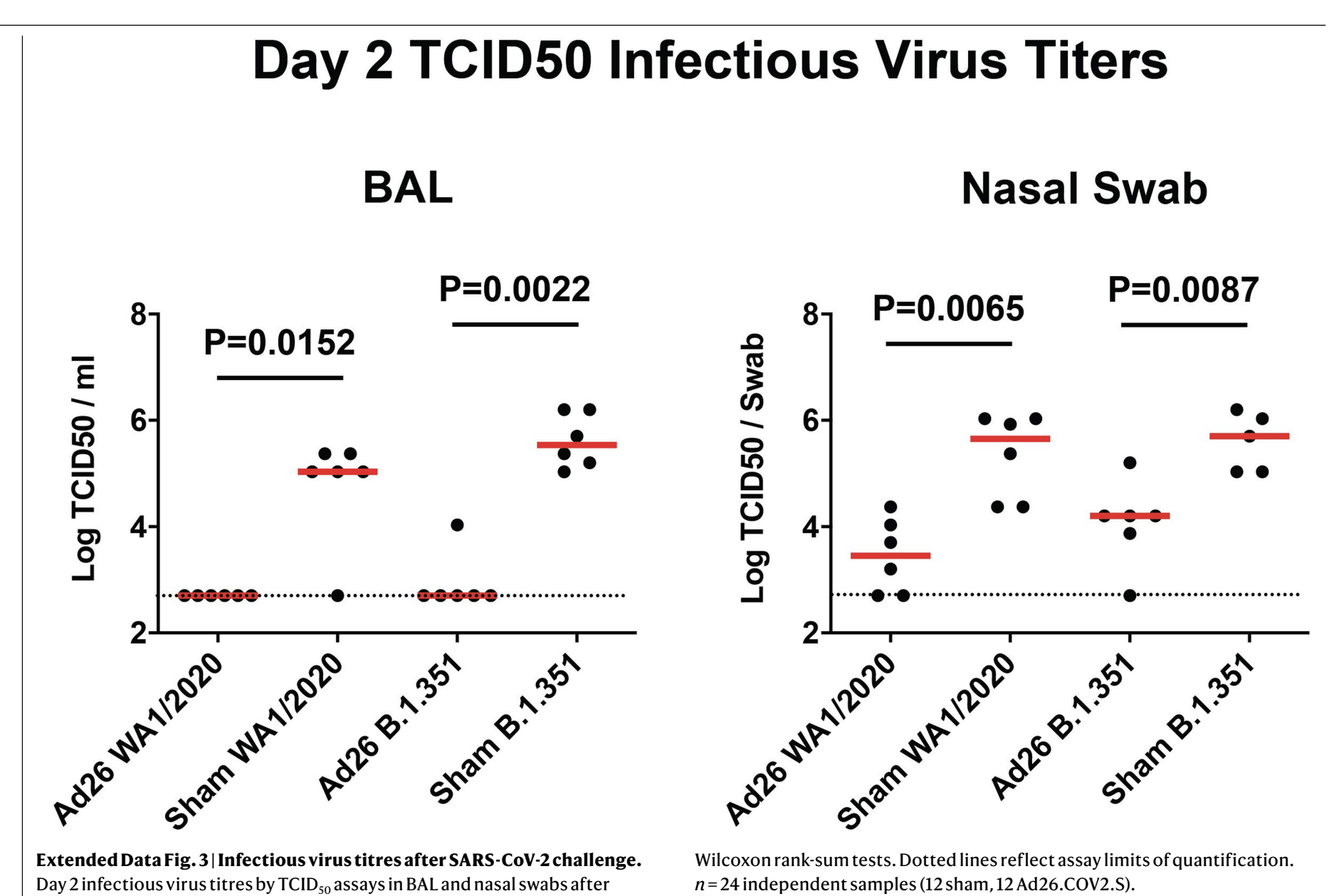




\section{Article}

a
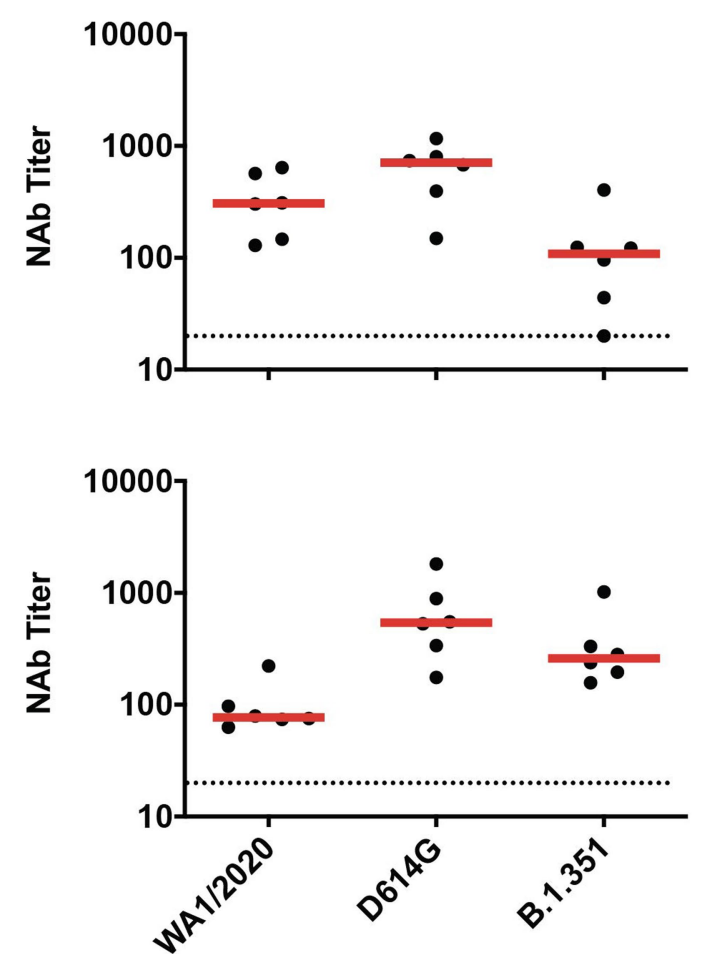

b
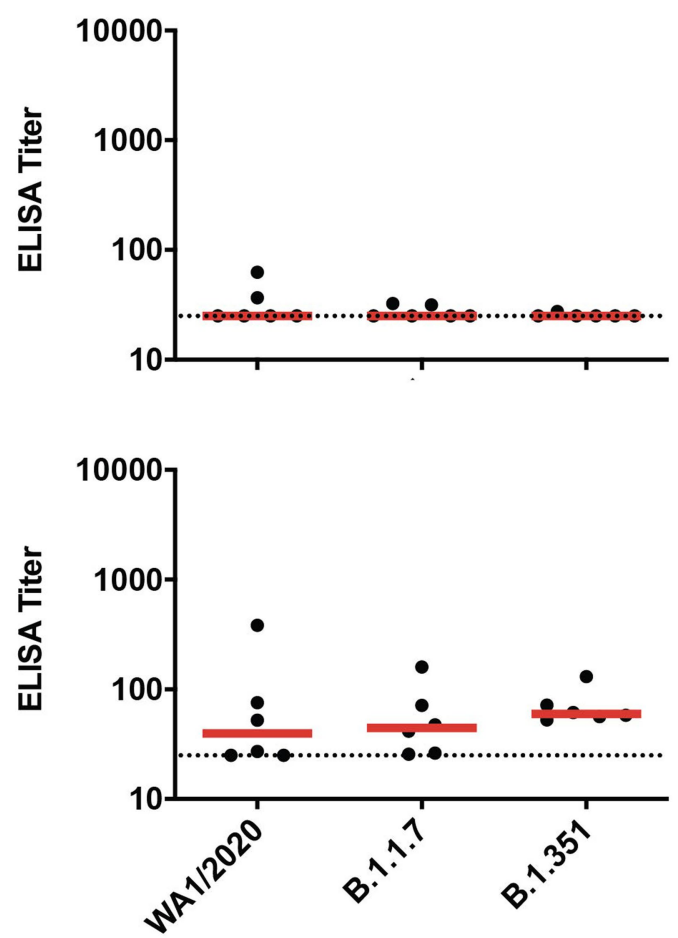

Extended Data Fig. 4 | Binding and neutralizing antibody responses in challenged rhesus macaques. a, b, Pseudovirus neutralizing antibody assays against the SARS-CoV-2 WA1/2020, D614G, and B.1.351 variants were assessed (a) and RBD-specific binding antibody responses against the SARS-CoV-2 WA1/2020, B.1.1.7, and B.1.351 variants were assessed by ELISA (b) on day 10
Ad26.COV2.S

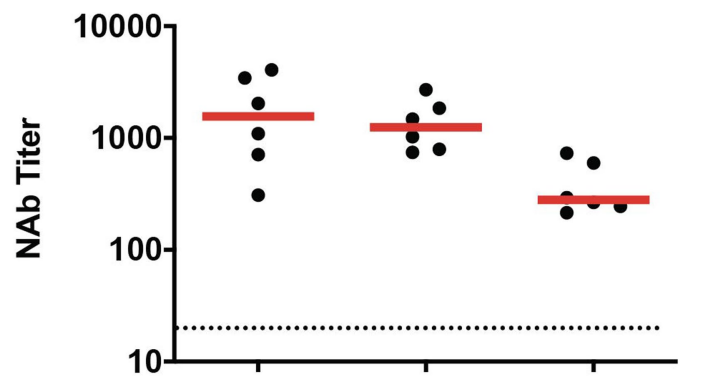

WA1/2020

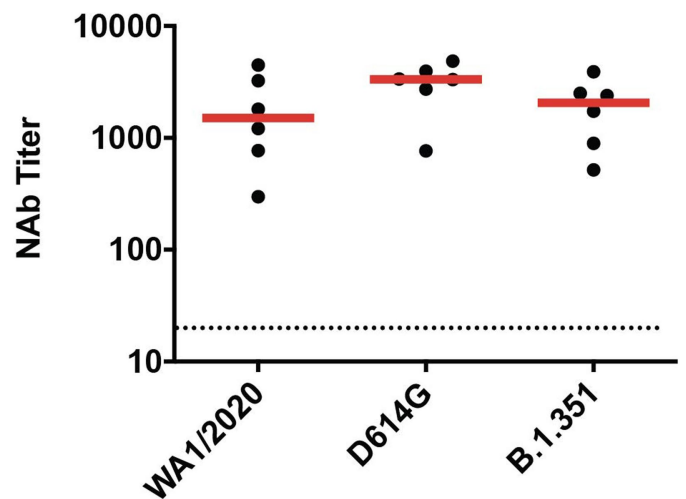

B.1.351
Ad26.COV2.S

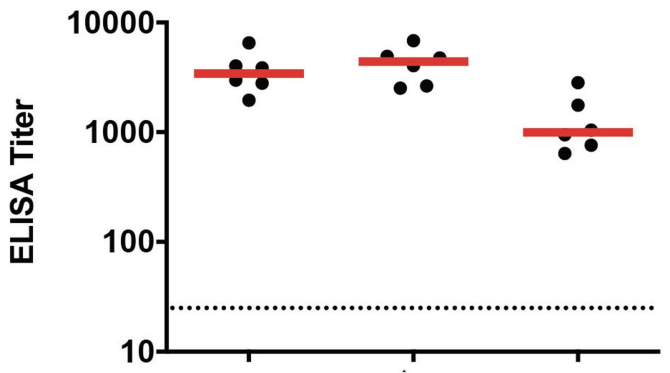

WA1/2020

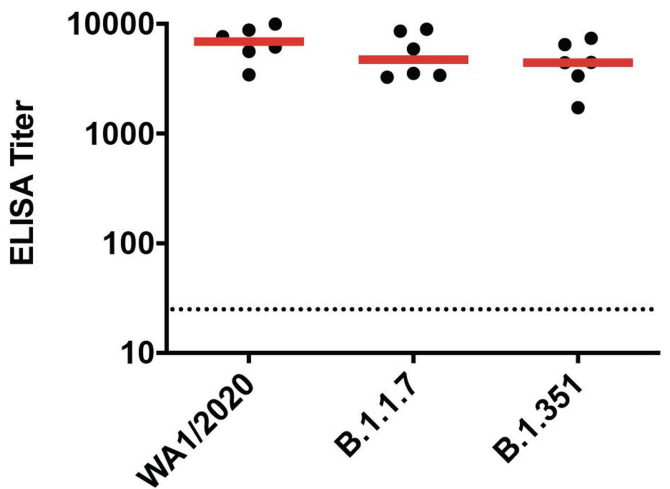

B.1.351

after challenge in macaques that received a single immunization of sham vaccine or $5 \times 10^{10} \mathrm{viral}$ particles of Ad26.COV2.S. Macaques that were challenged with WA1/2020 or B.1.351 are shown in separate graphs. Horizontal red bars reflect median responses. Dotted lines reflect assay limits of quantification. $n=24$ independent samples (12 sham, 12 Ad26.COV2.S). 


\section{Sham}
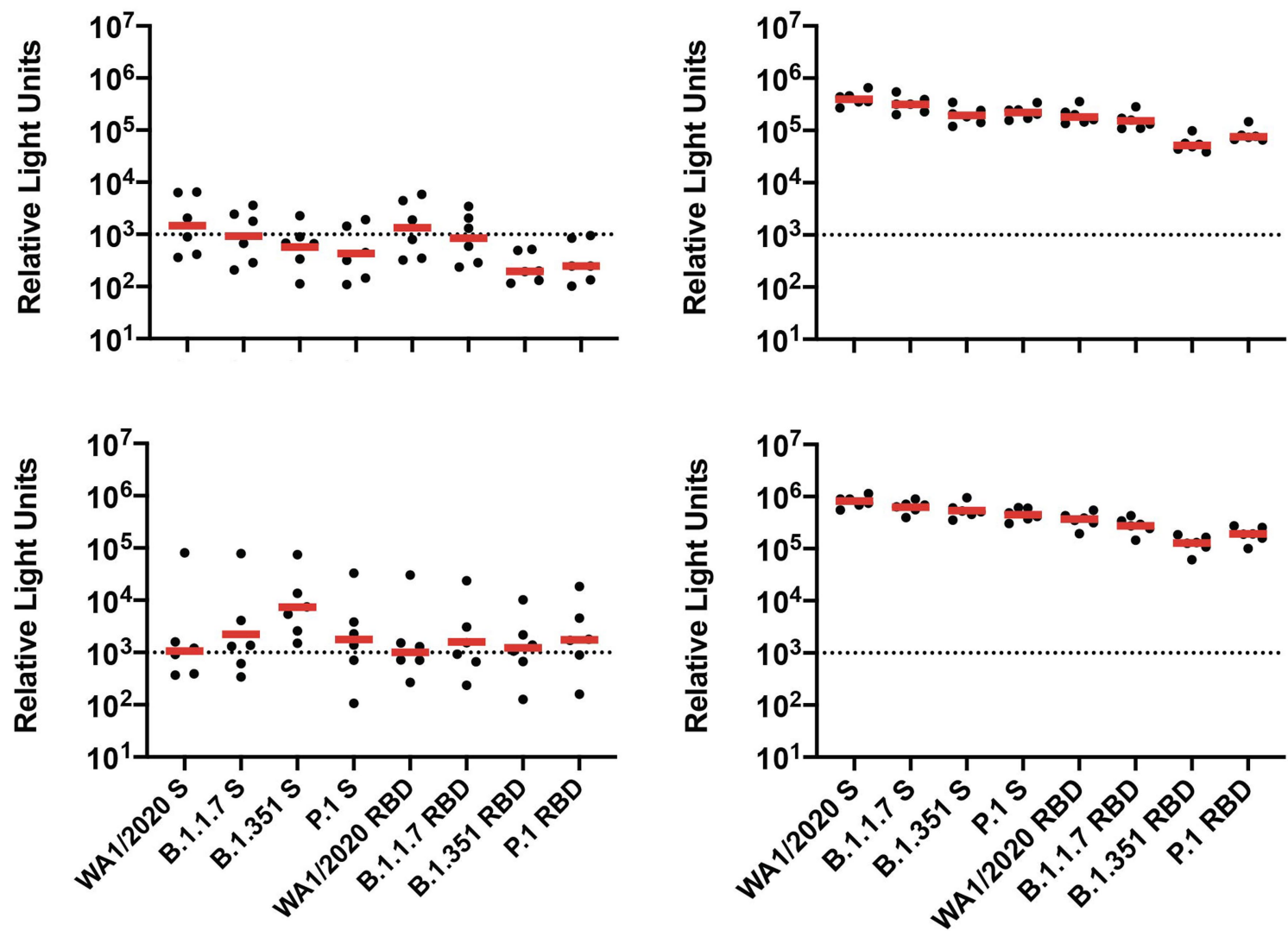

Macaques that were challenged with WA1/2020 or B.1.351 are shown in separate graphs. Horizontal red bars reflect median responses. Dotted lines reflect assay limit of quantification. $n=24$ independent samples (12 sham, 12 Ad26.COV2.S).

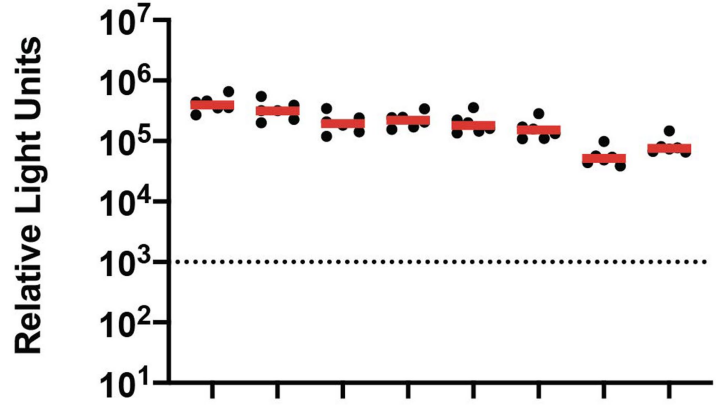

B.1.351
Extended Data Fig. 5 | Binding antibody responses in challenged rhesus macaques by ECLA.S- and RBD-specific binding antibody responses against the SARS-CoV-2 WA1/2020, B.1.1.7, B.1.351 and P.1 variants were assessed by ECLA on day 10 after challenge in macaques that received a single

immunization of sham vaccine or $5 \times 10^{10}$ viral particles of Ad26.COV2.S. 
Sham
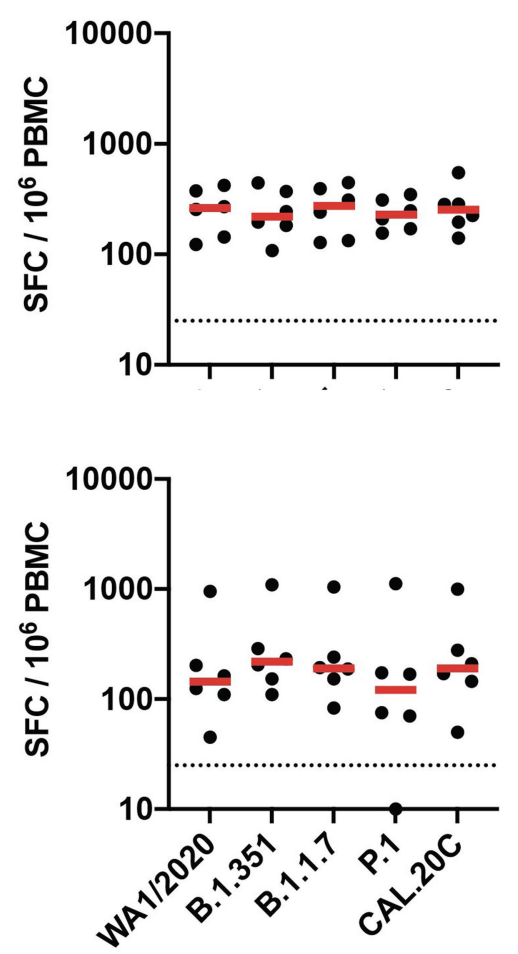

CD4
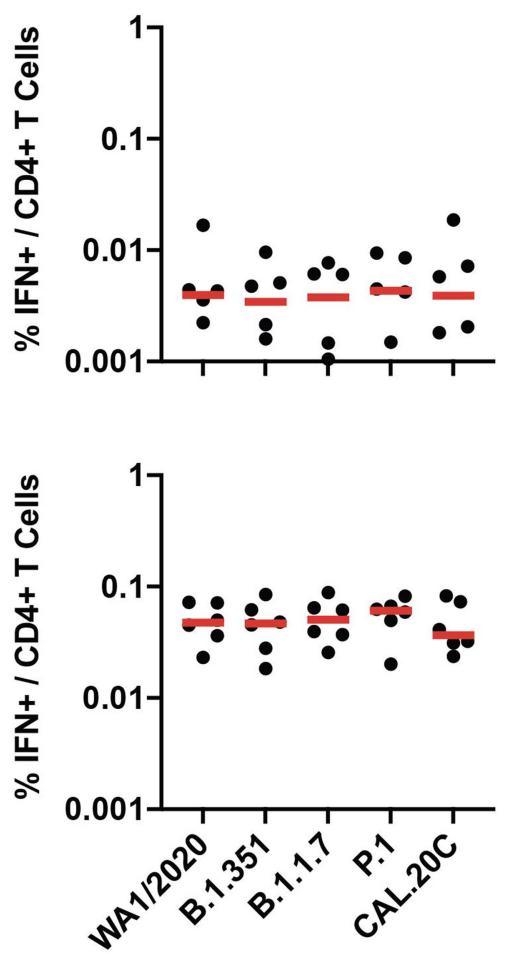

Extended Data Fig. $6 \mid \mathrm{T}$ cell responses in vaccinated rhesus macaques by ELISPOT assays. a, Cellular immune responses to pooled $S$ peptides were assessed by IFN $\gamma$ ELISPOT assays on day 10 after challenge to WA1/2020, B.1.351, B.1.1.7, P.1 and CAL.20C variants. b, $\mathrm{CD}^{+}{ }^{+}$and $\mathrm{CD} 8^{+} \mathrm{T}$ cell responses to
Ad26.COV2.S
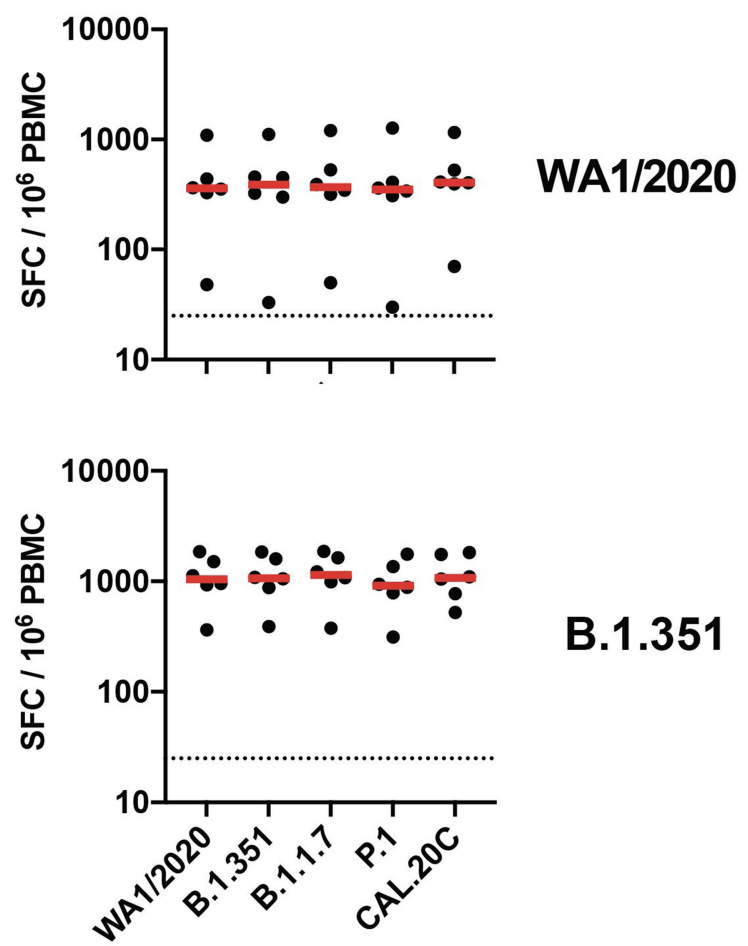

B.1.351

CD8

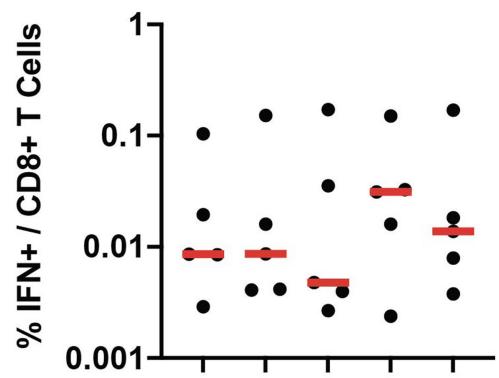

WA1/2020

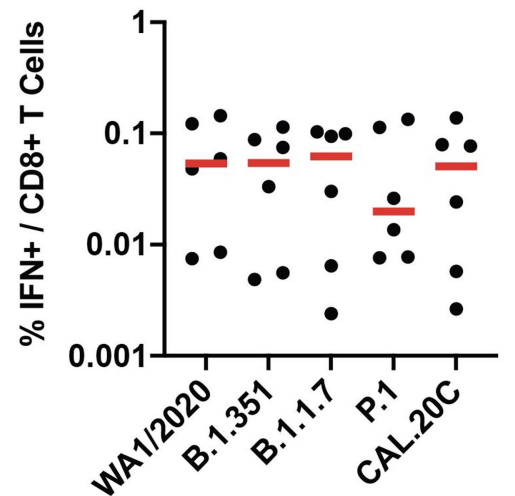

B.1.351

pooled $\mathrm{S}$ peptides were assessed by IFN $\gamma$ intracellular cytokine staining assays on day 10 after challenge to WA1/2020, B.1.351, B.1.1.7, P.1 and CAL.20C

variants. Horizontal red bars reflect median responses. $n=24$ independent samples (12 sham,12 Ad26.COV2.S). 

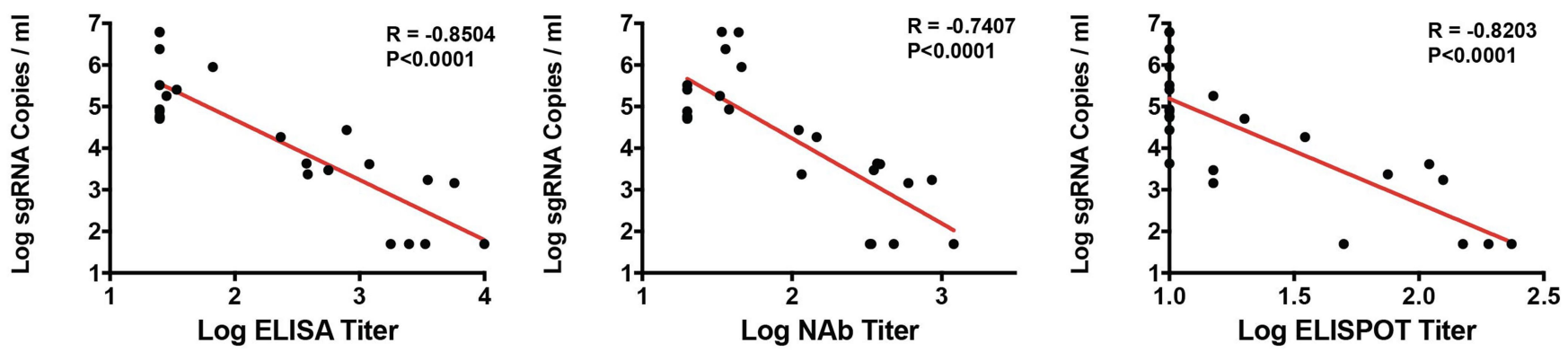

b
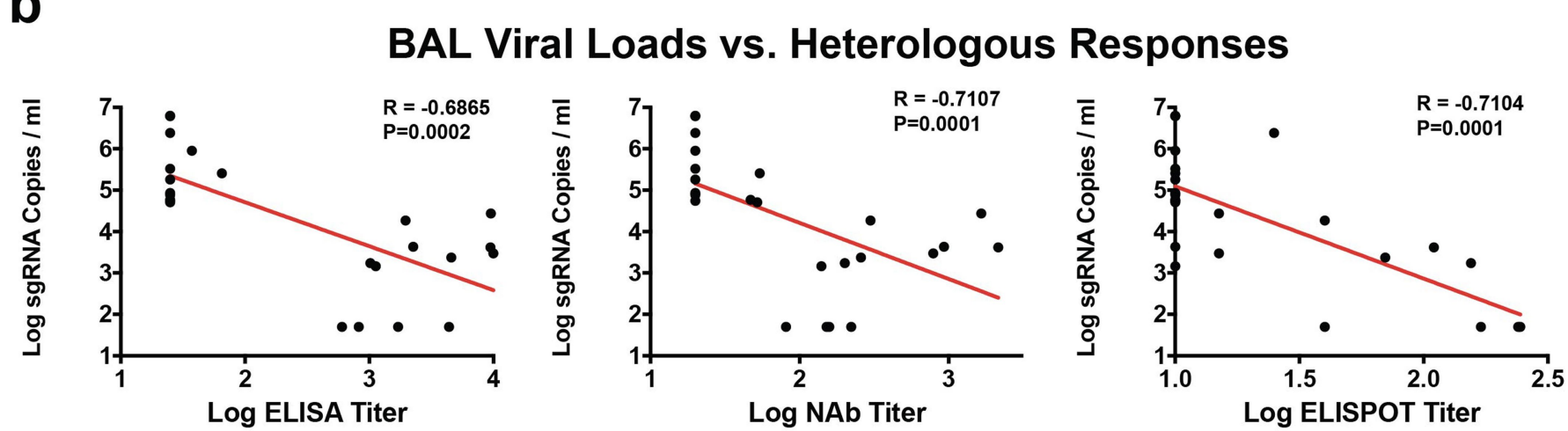

Extended Data Fig. 7 | Correlates of protection in BAL. a, b, Correlations of $\log$ (peak sgRNA copies per $\mathrm{ml}$ ) in BAL after challenge versus log-transformed ELISA titres, neutralizing antibody titres or ELISPOT responses to the

week 6 after vaccination. Red lines reflect the best linear fit relationship homologous (a) or heterologous (b) challenge virus (WA1/2020, B.1.351) at between these variables. $P$ and $R$ values reflect two-sided Spearman rank-correlation tests. 


\section{Article}

\section{a}

\section{Nasal Swab Viral Loads vs. Homologous Responses}
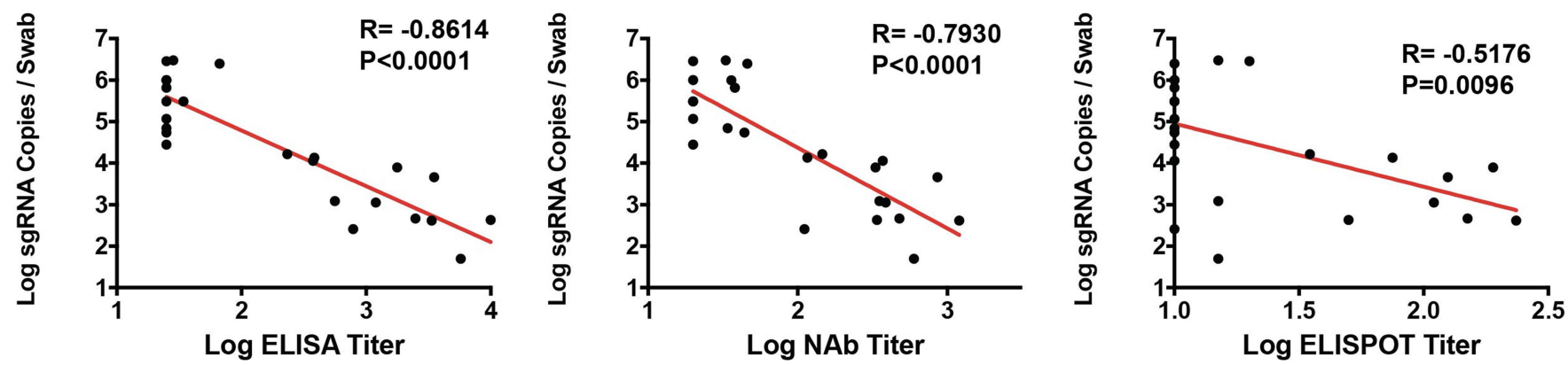

b

\section{Nasal Swab Viral Loads vs. Heterologous Responses}

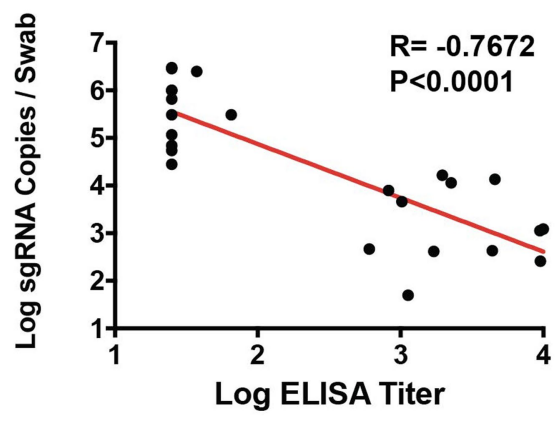

Extended Data Fig. 8 | Correlates of protection in nasal swabs.

a, b, Correlations of $\log$ (peak sgRNA copies per $\mathrm{ml}$ ) in nasal swabs after challenge versus log-transformed ELISA titres, neutralizing antibody titres or ELISPOT responses to the homologous (a) or heterologous (b) challenge virus

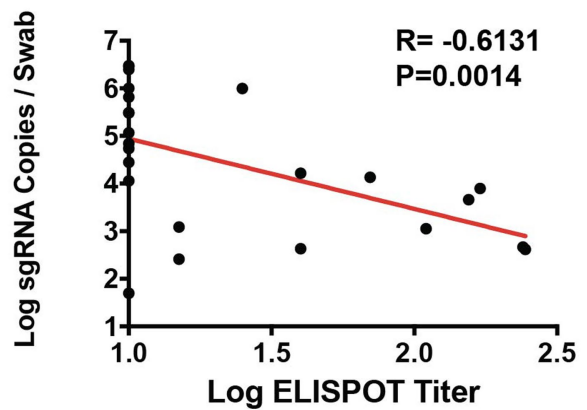

(WA1/2020, B.1.351) at week 6 after vaccination. Red lines reflect the best linear fit relationship between these variables. $P$ and $R$ values reflect two-sided Spearman rank-correlation tests. 


\section{Sham}

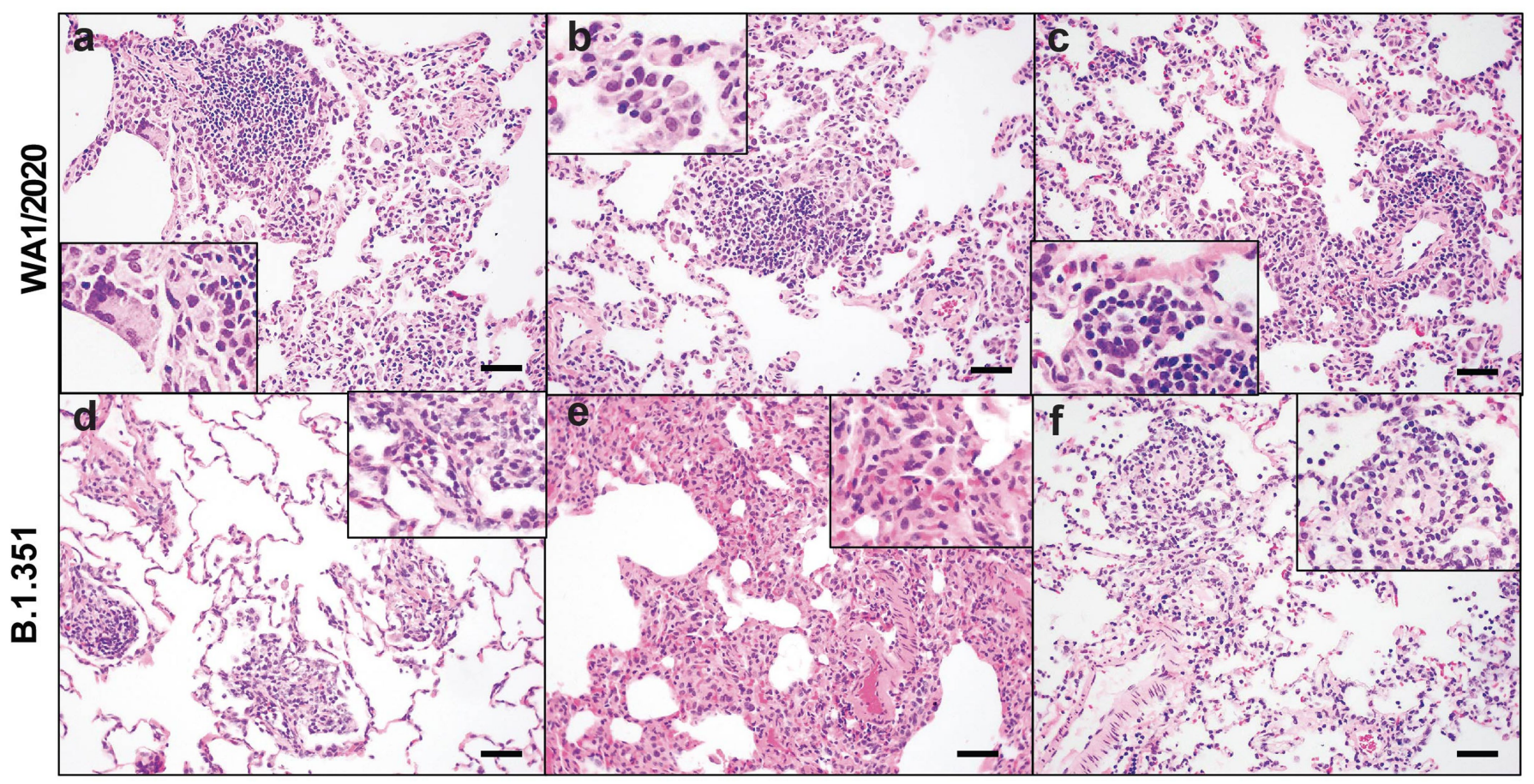

Extended Data Fig. 9 |Representative histopathology in sham control macaques after SARS-CoV-2 challenge. a-f, Locally extensive moderate-tosevere lesions were observed in sham control macaques challenged with WA1/2020 (a-c) or B.1.351 (d-f), on day 10 after challenge. a, Syncytia,

lymphoid proliferation and locally extensive interstitial inflammation.b, Type II pneumocyte hyperplasia and lymphoid proliferation. c, Perivascular alveolar infiltrates and interstitial inflammation. d, Alveolar macrophage infiltrates. e, Severe mononuclear alveolar infiltrates and pneumocyte hyperplasia. f, Perivascular infiltrates and interstitial inflammation. At least eight tissues were assessed per macaques. Haematoxylin and eosin staining. Scale bars, $20 \mu \mathrm{m}$. 


\section{Ad26.COV2.S}

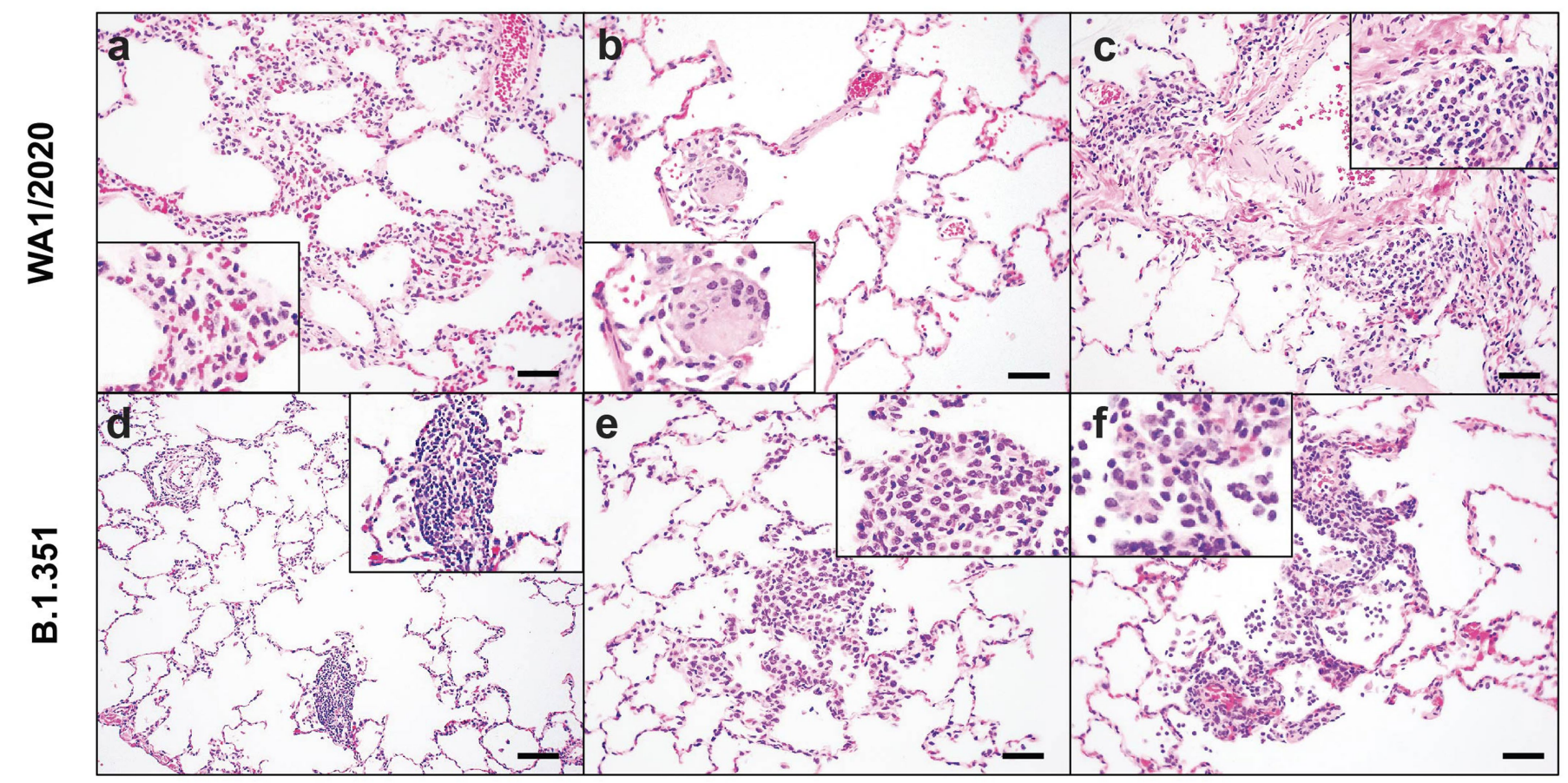

Extended Data Fig. 10 | Representative histopathology in Ad26. COV2.S-vaccinated macaques after SARS-CoV-2 challenge. a-f, Focal minimal-to-mild lesions were observed in Ad26.COV2.S-vaccinated macaques challenged with WA1/2020 (a-c) or B.1.351 (d-f), on day 10 after challenge. a, Interstitial inflammation. b, Syncytia. c, Perivascular neutrophilic infiltrates. d, Perivascular mononuclear inflammation. e, Type II pneumocyte hyperplasia. f, Alveolar macrophage infiltrates. At least eight tissues were assessed per macaque. Haematoxylin and eosin staining. Scale bar, $20 \mu \mathrm{m}$. 


\section{Reporting Summary}

Nature Research wishes to improve the reproducibility of the work that we publish. This form provides structure for consistency and transparency in reporting. For further information on Nature Research policies, see our Editorial Policies and the Editorial Policy Checklist.

\section{Statistics}

For all statistical analyses, confirm that the following items are present in the figure legend, table legend, main text, or Methods section.

n/a Confirmed

$\square$ The exact sample size $(n)$ for each experimental group/condition, given as a discrete number and unit of measurement

$\square$ A statement on whether measurements were taken from distinct samples or whether the same sample was measured repeatedly

$\triangle$ The statistical test(s) used AND whether they are one- or two-sided

Only common tests should be described solely by name; describe more complex techniques in the Methods section.

$\bigotimes$ A description of all covariates tested

$\square$ A description of any assumptions or corrections, such as tests of normality and adjustment for multiple comparisons

$\triangle$ A full description of the statistical parameters including central tendency (e.g. means) or other basic estimates (e.g. regression coefficient)

AND variation (e.g. standard deviation) or associated estimates of uncertainty (e.g. confidence intervals)

$\varnothing$ For null hypothesis testing, the test statistic (e.g. $F, t, r$ ) with confidence intervals, effect sizes, degrees of freedom and $P$ value noted

Give $P$ values as exact values whenever suitable.

$\bigotimes \square$ For Bayesian analysis, information on the choice of priors and Markov chain Monte Carlo settings

$\bigotimes \square$ For hierarchical and complex designs, identification of the appropriate level for tests and full reporting of outcomes

$\bigotimes \square$ Estimates of effect sizes (e.g. Cohen's $d$, Pearson's $r$ ), indicating how they were calculated

Our web collection on statistics for biologists contains articles on many of the points above.

\section{Software and code}

Policy information about availability of computer code

Data collection QuantStudio 6 was used to collect sgRNA data.

Data analysis Analysis of virologic and immunologic data was performed using GraphPad Prism 8.4.2 (GraphPad Software). Flow cytometry data was analyzed with FlowJo v9.9.

For manuscripts utilizing custom algorithms or software that are central to the research but not yet described in published literature, software must be made available to editors and reviewers. We strongly encourage code deposition in a community repository (e.g. GitHub). See the Nature Research guidelines for submitting code \& software for further information.

\section{Data}

Policy information about availability of data

All manuscripts must include a data availability statement. This statement should provide the following information, where applicable:

- Accession codes, unique identifiers, or web links for publicly available datasets

- A list of figures that have associated raw data

- A description of any restrictions on data availability

All data are available in the manuscript or the supplementary material. 


\section{Field-specific reporting}

Please select the one below that is the best fit for your research. If you are not sure, read the appropriate sections before making your selection. $\bigotimes$ Life sciences $\quad \square$ Behavioural \& social sciences $\quad \square$ Ecological, evolutionary \& environmental sciences

For a reference copy of the document with all sections, see nature.com/documents/nr-reporting-summary-flat.pdf

\section{Life sciences study design}

All studies must disclose on these points even when the disclosure is negative.

Sample size Sample size includes N=24 vaccinated animals ( $N=6$ animals/group; Mercado et al Nature 2020). Based on our experience with SARS-CoV-2 in rhesus macaques, this sample size can differentiate large differences in protective efficacy compared with the sham controls.

Data exclusions No data were excluded.

Replication Virologic and immunologic measures were performed in duplicate. Technical replicates were minimally different. All attempts at replication were successful.

Randomization Animals were balanced for age and gender and otherwise randomly allocated to groups.

Blinding All immunologic and virologic assays were performed blinded.

\section{Reporting for specific materials, systems and methods}

We require information from authors about some types of materials, experimental systems and methods used in many studies. Here, indicate whether each material, system or method listed is relevant to your study. If you are not sure if a list item applies to your research, read the appropriate section before selecting a response.

Materials \& experimental systems

\begin{tabular}{l|l}
\hline$n / a$ & Involved in the study \\
\hline & $\square$ Antibodies \\
$\square$ & $\square$ Animals and other organisms \\
$\square$ & $\square$ Clinical data \\
$\square$ & $\square$ Dual
\end{tabular}

\begin{tabular}{l|l}
\multicolumn{2}{l}{ Methods } \\
\hline n/a & Involved in the study \\
\hline & $\square$ ChIP-seq \\
$\square$ & $\bigotimes$ Flow cytometry \\
$\square$ & $\square$ MRI-based neuroimaging
\end{tabular}

\section{Antibodies}

Antibodies used

For ELISA and ELISPOT assays anti-macaque IgG HRP (NIH NHP Reagent Program), rabbit polyclonal anti-human IFN-y (U-Cytech); for ICS assays mAbs against CD279 (clone EH12.1, BB700), CD38 (clone OKT10, PE), CD28 (clone 28.2, PE CY5), CD4 (clone L200, BV510), CD45 (clone D058-1283, BUV615), CD95 (clone DX2, BUV737), CD8 (clone SK1, BUV805), Ki67 (clone B56, FITC), CD69 (clone

TP1.55.3, ECD), IL10 (clone JES3-9D7, PE CY7), IL13 (clone JES10-5A2, BV421), TNF- $\alpha$ (clone Mab11, BV650), IL4 (clone MP4-25D2, BV711), IFN- $\gamma$ (clone B27; BUV395), IL2 (clone MQ1-17H12, APC), CD3 (clone SP34.2, Alexa 700) (BD); for 800CW-conjugated goatanti-human secondary antibody (Li-COR); anti-rhesus IgG1, IgG2, IgG3, IgA, IgM (NIH NHP Reagent Program); tertiary goat anti-mouse IgG-PE antibody (Southern Biotech), anti-CD107a (PE-Cy7, BD), anti-CD56 (PE-Cy7, BD), anti-MIP-1B (PE, BD), mouse anti-human IFNy monoclonal antibody (BD), Streptavidin-alkaline phosphatase antibody (Southern Biotech), CD49d (BD), sulfo-tagged anti-human IgG (MesoScale Discovery).

Validation all mAbs used according to manufacturer's instructions and were titrated prior to use

\section{Eukaryotic cell lines}

Policy information about cell lines

Cell line source(s)

None

Authentication

Commerically purchased (ATCC) and evaluated in control experiments prior to use

Mycoplasma contamination

Negative for mycoplasma 
Commonly misidentified lines

(See ICLAC register)
None were utilized

\section{Animals and other organisms}

Policy information about studies involving animals; ARRIVE guidelines recommended for reporting animal research

Laboratory animals 24 outbred Indian-origin adult male and female rhesus macaques (Macaca mulatta), 3-11 years old

Wild animals None

Field-collected samples None

Ethics oversight Bioqual IACUC

Note that full information on the approval of the study protocol must also be provided in the manuscript.

\section{Flow Cytometry}

\section{Plots}

Confirm that:

$\bigotimes$ The axis labels state the marker and fluorochrome used (e.g. CD4-FITC).

ХThe axis scales are clearly visible. Include numbers along axes only for bottom left plot of group (a 'group' is an analysis of identical markers).

$\bigotimes$ All plots are contour plots with outliers or pseudocolor plots.

$\bigotimes$ A numerical value for number of cells or percentage (with statistics) is provided.

\section{Methodology}

Sample preparation

Instrument

Software

Cell population abundance

Gating strategy
Isolated PBMC

BD FACSymphony

FlowJo v9.9

No sorting was performed

See gating strategy in Supplementary Figure 1

$\bigotimes$ Tick this box to confirm that a figure exemplifying the gating strategy is provided in the Supplementary Information. 